\title{
Planar lattice subsets with minimal vertex boundary
}

\author{
Radhika Gupta* \\ Department of Mathematics \\ Temple University \\ PA, U.S.A. \\ radhikagupta.maths@gmail.com
}

\author{
Alex Margolis ${ }^{\ddagger}$ \\ Department of Mathematics \\ Vanderbilt University \\ TN, U.S.A. \\ alexander.margolis@vanderbilt.edu
}

\author{
Ivan Levcovitz ${ }^{\dagger}$ \\ Department of Mathematics \\ Tufts University \\ MA, U.S.A. \\ ivan.levcovitz@tufts.edu
}

Submitted: Dec 1, 2020; Accepted: Aug 31, 2021; Published: Sep 24, 2021

(C) The authors. Released under the CC BY license (International 4.0).

\begin{abstract}
A subset of vertices of a graph is minimal if, within all subsets of the same size, its vertex boundary is minimal. We give a complete, geometric characterization of minimal sets for the planar integer lattice $X$. Our characterization elucidates the structure of all minimal sets, and we are able to use it to obtain several applications. We show that the neighborhood of a minimal set is minimal. We characterize uniquely minimal sets of $X$ : those which are congruent to any other minimal set of the same size. We also classify all efficient sets of $X$ : those that have maximal size amongst all such sets with a fixed vertex boundary. We define and investigate the graph $\mathcal{G}$ of minimal sets whose vertices are congruence classes of minimal sets of $X$ and whose edges connect vertices which can be represented by minimal sets that differ by exactly one vertex. We prove that $\mathcal{G}$ has exactly one infinite component, has infinitely many isolated vertices and has bounded components of arbitrarily large size. Finally, we show that all minimal sets, except one, are connected.
\end{abstract}

Mathematics Subject Classifications: 05D99, 05B25

${ }^{*}$ Supported by Israel Science Foundation Grant 1026/15 and EPSRC grant EP/R042187/1 and would also like to thank Séphora Berrebi Scholarships.

${ }^{\dagger}$ Supported by the Israel Science Foundation and in part by a Technion fellowship.

¥Supported by the Israel Science Foundation Grant No. 1562/19.

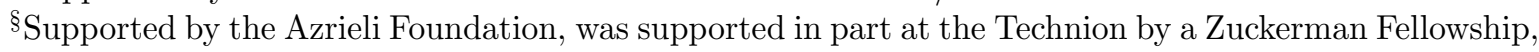
and was partially supported by NSF RTG grant \#1849190. 


\section{Introduction}

The classical isoperimetric problem can be stated as follows: amongst all closed curves in the plane with fixed length, characterize those that enclose the maximal area. The solution to this isoperimetric problem is the circle. By a simple scaling argument, this problem is easily seen to be equivalent to the following dual problem:

Problem 1. Amongst all closed curves in the plane that enclose a fixed area, characterize those that have minimal length.

The isoperimetric problem dates back to antiquity, as documented in Virgil's account of the founding of Carthage in the Aeneid. However, the first steps towards a solution of Problem 1 were given relatively recently by Steiner in the $19^{\text {th }}$ century. In this article, we give a solution to the discrete graph-theoretic analogue of Problem 1.

Discrete isoperimetric problems have been studied extensively in graph theory, and there are many applications in areas such as network design and the theory of error correcting codes [Har04, HLW06]. Given a graph $X$ with vertex set $V(X)$, the vertex boundary of $A \subset V(X)$ is defined by

$$
\partial A:=\{u \in V(X) \backslash A \mid \text { there exists } v \in A \text { such that }(u, v) \in E(X)\} .
$$

The vertex isoperimetric problem for a graph $X$ is the following:

Problem 2. Amongst all subsets of $V(X)$ with a fixed number of vertices, characterize those that have minimal size vertex boundary.

The sets that appear as solutions to Problem 2 are called minimal.

\section{The isoperimetric problem for the integer lattice in the plane}

In this article we study the graph $X=\mathbb{Z}_{\ell_{1}}^{2}$ with vertex set $X^{0}=\mathbb{Z}^{2}$ and edges connecting all pairs of vertices $\ell_{1}$-distance one apart. A nested sequence of minimal sets for $X$ is given by Wang-Wang [WW77].

Our approach differs from the usual one of finding a sequence of minimal sets, in that we give a geometric characterization of every minimal set. While circles are the natural geometric solution to Problem 1, there can be many different congruence classes of minimal sets in $X$ of a given size and our result exactly describes these solutions. This approach lets us prove many applications that allow us to better understand the collection of all minimal sets.

Before describing our results, we first establish some notation. We consider subsets of $X^{0}$ up to the following natural equivalence relation: we say two subsets $A, B \subset X^{0}$ are congruent if there is a graph automorphism $\phi$ of $X$ such that $\phi(A)=B$. It is clear that if $A$ and $B$ are congruent, then $A$ is minimal if and only if $B$ is minimal.

Given natural numbers $\alpha, \beta \in \mathbb{N}$, we define $B(\alpha, \beta)$ to be the set of vertices $(x, y) \in X^{0}$ that satisfy $0 \leqslant y-x \leqslant \alpha$ and $0 \leqslant y+x \leqslant \beta$. Similarly, given even integers $\alpha, \beta \in \mathbb{N}$, we define $\hat{B}(\alpha, \beta)$ to be the set of vertices $(x, y) \in X^{0}$ that satisfy $0 \leqslant y-x \leqslant \alpha$ and 
$-1 \leqslant y+x \leqslant \beta-1$. A box is a non-empty subset of $X^{0}$ that is congruent to either $B(\alpha, \beta)$ or $\hat{B}(\alpha, \beta)$ for some $\alpha, \beta$. The enclosing box of a set $A$, denoted enc $(A)$, is the smallest box containing $A$. Examples of sets and their enclosing boxes are shown in Figure 1.1.

Since boxes are parametrized by numbers $\alpha, \beta \in \mathbb{N}$, it is easier to determine whether a box is minimal than it is to determine whether an arbitrary set of vertices is minimal. Therefore, our broad strategy in solving Problem 2 is to compare an arbitrary set to its enclosing box. We show that for minimal sets, the enclosing box can be obtained by "saturating" a set, i.e. by adding vertices that do not increase the boundary. In the course of our proof, we classify precisely which boxes are minimal, see Remark 5.14.

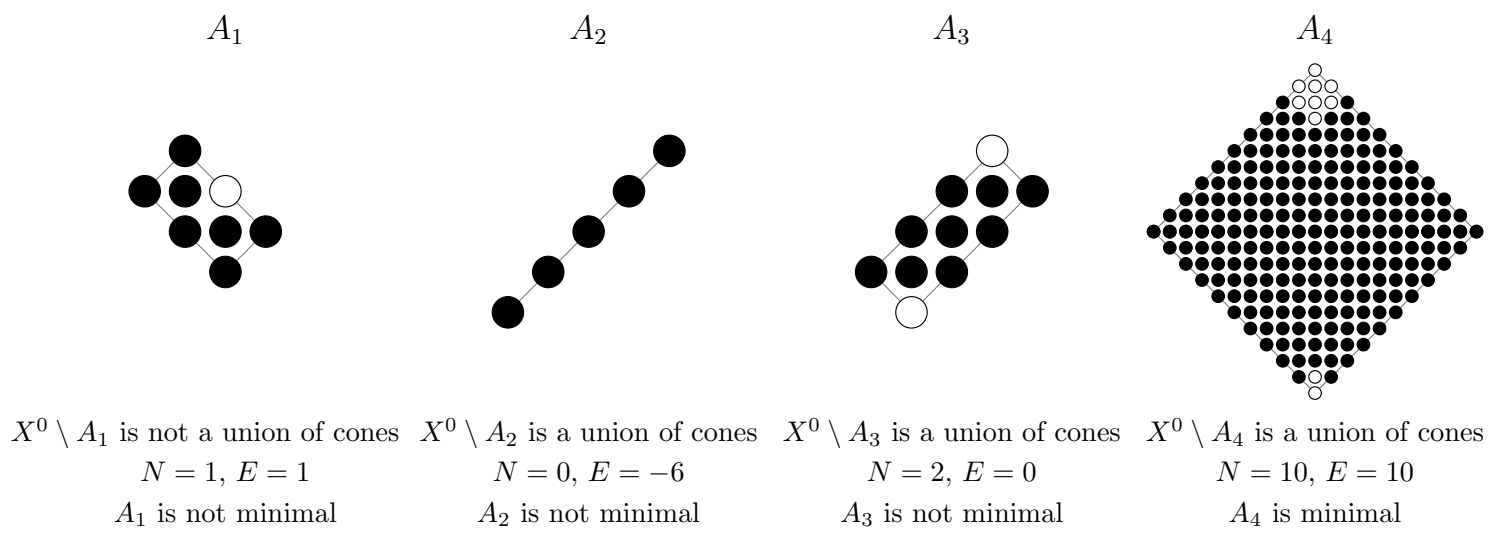

Figure 1.1: Examples of sets $A_{i} \subset X^{0}$ and their enclosing boxes. Black vertices are contained in $A_{i}$ and white vertices are contained in enc $\left(A_{i}\right) \backslash A_{i}$.

A cone is a subset of $X^{0}$ congruent to the set $\{(x, y) \mid y-x \geqslant 0, y+x \geqslant 0\}$. As a precursor to our main result, we give a necessary condition for minimality: if a set $A$ is minimal, then its complement $X^{0} \backslash A$ is a union of cones and furthermore, its enclosing box enc $(A)$ is also minimal. Although this is far from a complete classification of minimal sets - which we give in Theorem A - it demonstrates the important role of the enclosing box in determining minimality.

If $A_{1}$ is the set shown in Figure 1.1, its complement $X^{0} \backslash A_{1}$ is not a union of cones. This follows as any cone containing the white vertex in enc $\left(A_{1}\right) \backslash A_{1}$ must also contain a vertex of $A_{1}$, and so $X^{0} \backslash A_{1}$ is not a union of cones. Thus $A_{1}$ is not minimal by the preceding necessary condition. Similarly, $A_{2}$ can be seen not to be minimal since its enclosing box enc $\left(A_{2}\right)$ is not minimal. However, to say whether or not $A_{3}$ and $A_{4}$ are minimal is a slightly more delicate matter, since both $A_{3}$ and $A_{4}$ satisfy the preceding necessary condition for minimality. To see why $A_{3}$ is not minimal and $A_{4}$ is, we use a numerical invariant of a box called its excess.

Denoted $\operatorname{Ex}(B)$, the excess of a box $B$ measures how much larger a box is than the smallest minimal set $A$ with $|\partial A|=|\partial B|$; see Definition 4.1. In particular, the excess of a box is non-negative if and only if the box is minimal. An explicit formula for the excess of a box is given in Theorem 5.13. In particular, this implies a box $B(\alpha, \beta)$ is minimal if it satisfies $|\beta-\alpha| \leqslant 1+\min (\sqrt{4 \alpha+1}, \sqrt{4 \beta+1})$, and this bound is close to tight. Thus, a box is minimal if it is sufficiently close to being a square. 
Our main theorem, stated below, gives two related characterizations of minimal sets in terms of their enclosing boxes.

Theorem A (Theorem 7.8). Let $A \subset X^{0}$ with $N:=|\operatorname{enc}(A) \backslash A|$ and $E:=\operatorname{Ex}(\operatorname{enc}(A))$. Then the following are equivalent:

1. A is minimal;

2. $|\partial A|=|\partial(\operatorname{enc}(A))|$ and $N \leqslant E$;

3. $X^{0} \backslash A$ is a union of cones and $N \leqslant E$.

Going back to the examples in Figure 1.1, a straightforward application of Theorem 5.13 - the formula for the excess of a box - tells us that $A_{3}$ does not satisfy $N \leqslant E$, but $A_{4}$ does. Since $X^{0} \backslash A_{3}$ and $X^{0} \backslash A_{4}$ are both unions of cones, Theorem A can be used to deduce that $A_{3}$ is not minimal and $A_{4}$ is minimal.

\section{Applications}

If $A \subset X^{0}$, then the neighborhood of $A$, denoted $N(A)$, is the set $N(A)=A \cup \partial A$. The neighborhood of a Wang-Wang set is a Wang-Wang set; see Figure 2.1. Hence, neighborhoods of the specific minimal sets exhibited by Wang-Wang for $\left(\mathbb{Z}^{d}, \ell_{1}\right)$ are also minimal. We use our characterization of all minimal sets given in Theorem 7.8 to show that minimality in $X$ is preserved when passing to neighborhoods.

Theorem B. If $A \subset X^{0}$ is a minimal set, then $N(A)$ is a minimal set.

A natural question to consider is whether minimal sets of a fixed size are unique up to congruence. More formally, $A \subset X^{0}$ is uniquely minimal if $A$ is minimal and any minimal set containing the same number of vertices as $A$ is congruent to $A$. We completely classify uniquely minimal sets:

Theorem C (Theorem 8.8). A subset of $X^{0}$ is uniquely minimal if and only if it is congruent to either $B(2 n, 2 n)$ or $B(n, n+1)$ for some $n \in \mathbb{N}$.

As well as understanding individual minimal sets, we also want to understand the structure of the collection of all minimal sets. To do this, we initiate the study of the graph $\mathcal{G}$ of minimal sets. Vertices of $\mathcal{G}$ are congruence classes of minimal sets. Two vertices $v$ and $w$ in $\mathcal{G}$ are joined by an edge if there exist representative minimal sets $A \in v$ and $B \in w$ whose symmetric difference has size one. The graph $\mathcal{G}$ has a natural grading corresponding to the sizes of representative minimal sets. The induced subgraphs of $\mathcal{G}$ containing all congruence classes of minimal sets of size at most 10 and 41 are shown in Figures 1.2 and 1.3 respectively.

We exhibit the following features of $\mathcal{G}$ :

Theorem D. Let $\mathcal{G}$ be the graph of minimal sets. Then:

1. $\mathcal{G}$ contains a single infinite component (Corollary 8.9); 


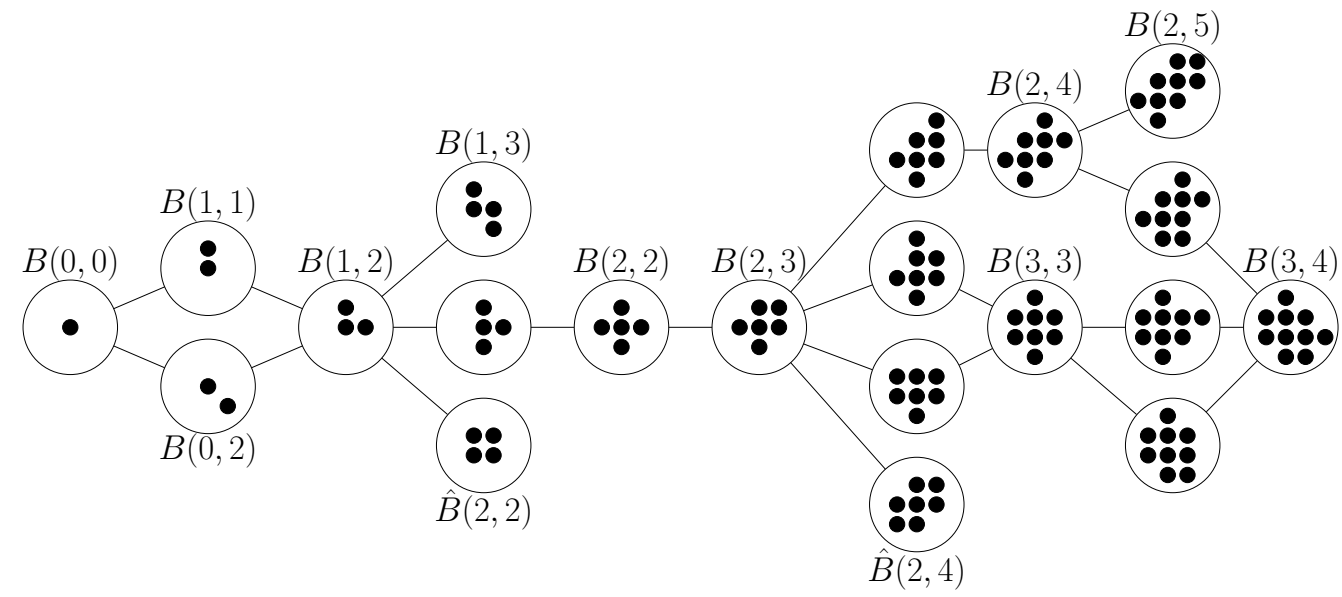

Figure 1.2: The induced subgraph of $\mathcal{G}$ containing congruence classes of minimal sets of size at most 10. All boxes are labelled using Notation 5.5.

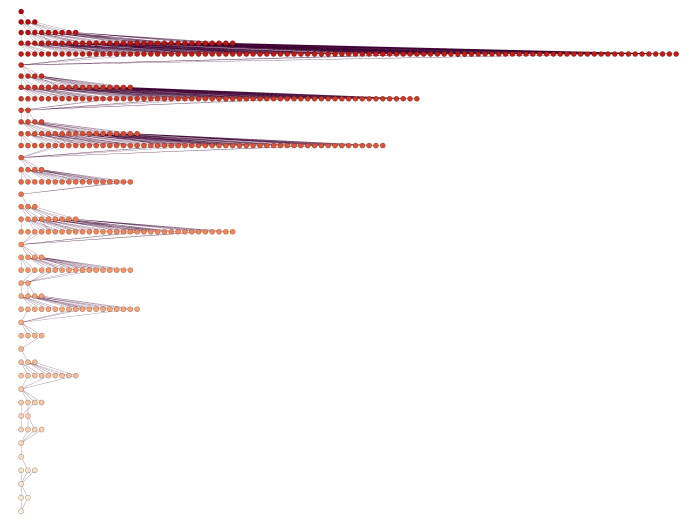

(a) The induced subgraph of $\mathcal{G}$ of congruence classes of minimal sets of size up to 41 . The graded structure of the graph is shown where vertices representing sets of larger sizes appear above and are shaded with a darker color than those representing smaller sizes.

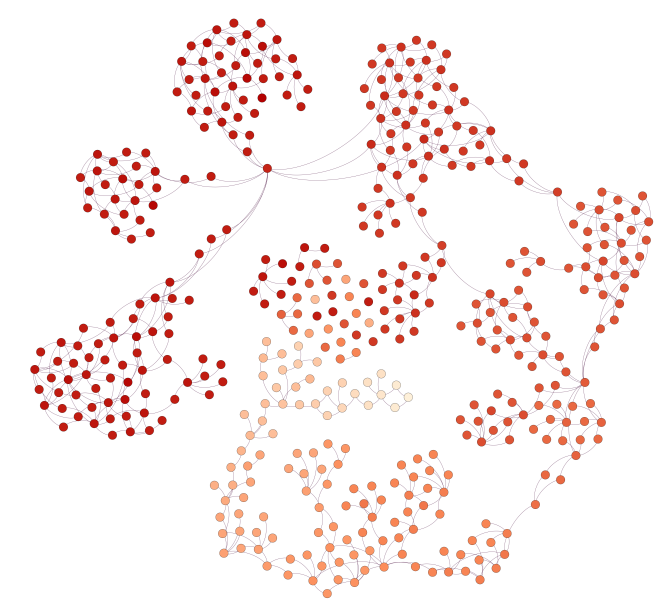

(b) The same graph as on the left displayed here in a different layout. Isolated vertices and finite size components are towards the center of the graph. The darkness of the shading of vertices is still proportional to the size of the corresponding sets.

Figure 1.3 
2. $\mathcal{G}$ contains finite components of arbitrarily large height (Theorem 8.11), where the height of a component is the maximal length of a nested sequence of minimal sets in it;

3. $\mathcal{G}$ contains infinitely many isolated vertices (Theorem 8.11).

We note that the infinite sequence of nested minimal sets constructed by Wang-Wang is contained in the unique infinite component of $\mathcal{G}$.

At the beginning of the introduction we mentioned two equivalent formulations of the isoperimetric problem in the Euclidean plane: maximizing the area enclosed by a curve of fixed length, or minimising the length of a curve enclosing a fixed area. We thus consider the following discrete isoperimetric problem dual to Problem 2:

Problem 3. Amongst all subsets of $X^{0}$ with a fixed vertex boundary, characterize those with maximal size.

We say a subset of $X^{0}$ is an efficient set if it is a solution to Problem 3. Every efficient set is minimal (see Lemma 8.1), but, in contrast with the continuous case, not every minimal set is efficient. We give an explicit solution to Problem 3:

Theorem E (Lemma 8.3). A subset of $X^{0}$ is efficient if and only if it is congruent to either $B(n, n), B(n, n+1)$ or $B(2 n, 2 n+2)$ for some $n \in \mathbb{N}$.

While writing this article, we learned that Theorem E was essentially already known. Vainsencher-Bruckstein characterize sets that are both efficient and minimal, which they call Pareto optimal sets [VB08]. However, by Wang-Wang's result and an easy argument, it follows that efficient sets are always minimal, giving the above theorem. We note that our proof is independent of that of Vainsencher-Bruckstein.

Consider the partial order on the set of minimal sets, where $A \prec B$ if there exists a nested sequence $A_{0} \subset A_{1} \subset \cdots \subset A_{n}$ of minimal sets with $\left|A_{i+1}\right|=\left|A_{i}\right|+1, A_{0}$ congruent to $A$ and $A_{n}$ congruent to $B$. In other words, there is a strictly grade-increasing path in $\mathcal{G}$ from $A$ to $B$. A minimal set is immortal if it lies in an infinite chain of this poset and is mortal otherwise. A minimal set is dead if it is maximal in this poset. We characterize mortal and dead sets:

Theorem F. Let $A \subset X^{0}$ be a minimal set. Then:

1. A is dead if and only if it is a box that is not efficient (Theorem 8.5).

2. A is mortal if and only if its enclosing box is dead (Proposition 8.6).

We note that by Theorem E we get an explicit characterization of mortal and dead sets in terms of box parametrizations.

A set $A \subset X^{0}$ is connected if its induced subgraph is connected. It can be seen in Figure 1.2 that the box $B(0,2)$ is minimal but is not connected. We show that, up to congruence, this is the only minimal set that is not connected. 
Theorem G (Theorem 7.1). A minimal set in $X$ is connected if and only if it is not congruent to $B(0,2)$.

\section{Other Related Works}

A complete solution to Problem 2 is known for very few graphs. Much of the literature has focused on exhibiting a sequence of minimal sets, i.e. a sequence $\left(A_{n}\right)$ where each $A_{n}$ is a minimal set consisting of exactly $n$ vertices. Finding such a sequence is NP-hard for a general graph (see [Har04]) and such sequences can typically be described only in special cases for graphs with an abundance of symmetry.

Harper exhibited a nested sequence of minimal sets for the $d$-dimensional hypercube $Q_{d}$, where $Q_{d}$ is a graph on the vertex set $\{0,1\}^{d}$ with an edge between a pair of binary strings that differ in a single coordinate [Har66]. This result was extended to $\left(P_{q}\right)^{d}$, the $d$-fold product of paths on $q$ vertices [Chv75, Mog83, BL91a]. For $\left(K_{q}\right)^{d}$, the $d$-fold product of complete graph on $q$ vertices, lower bounds and asymptotic solutions for the vertex isoperimetric problem are given in [Har99]. In constrast with $Q_{d}$, there is no nested sequence of minimal sets for $\left(K_{q}\right)^{d}$. The vertex isoperimetric problem on an $n$-dimensional even discrete torus was solved by [Rio98] and [Kar82] (see also [BL09]). More generally, in [BS02] the authors provide a general local-global principle to solve the vertex isoperimetric problem in all cartesian powers of a graph $G$ whenever $G$ and $G \times G$ admit a particular solution for the problem.

The isoperimetric problem has also been studied on infinite graphs, including integer lattices. Let $\mathbb{Z}_{\ell_{1}}^{d}$ (respectively $\mathbb{Z}_{\ell_{\infty}}^{d}$ ) be the graph on the vertex set $\mathbb{Z}^{d}$ where two vertices are joined by an edge if their $\ell_{1}$-distance (respectively $\ell_{\infty}$-distance) is 1 . As already mentioned, Wang-Wang [WW77] exhibit a nested sequence of minimal sets in $\mathbb{Z}_{\ell_{1}}^{d}$. Sieben gives a formula for the size of the vertex boundary of a minimal set of size $n$ in this graph under the assumption that such sets are connected [Sie08]. This is then used to analyze strategies for what are called polyomino achievement games. Radcliffe-Veomett obtain a nested sequence of minimal sets in $\mathbb{Z}_{\ell_{\infty}}^{d}$ [VR12].

The edge boundary of a subset of a graph is defined to be the set of edges that are incident to both a vertex of this subset and to a vertex outside this subset. The edge isoperimetric problem has also been well-studied for the various graphs mentioned above, namely, by [Har64, Lin64, Ber67, Har76] for $Q_{d}$, by [Lin64] for $\left(K_{q}\right)^{d}$ and [BL91b] for $\left(P_{q}\right)^{d}$ and $\mathbb{Z}_{\ell_{1}}^{d}$. For a useful survey on the edge isoperimetric problem on graphs see [Bez99]. Recently, [BE18] studied the edge isoperimetric problem for $\mathbb{Z}_{\ell_{\infty}}^{d}$ and $\mathbb{Z}^{d}$ with respect to any Cayley graph.

Many of these preceding results use a technique called compression or normalization that replaces a vertex set $A \subset V(X)$ with a set $c(A) \subset V(X)$ such that $|A|=|c(A)|$ and $|\partial A| \geqslant|\partial(c(A))|$; see [Har66]. Whilst this technique is well-suited to finding a sequence of minimal sets, it does not generally allow one to give a structural characterization of all minimal sets. 


\section{Outline}

In Section 2 we review the sequence of minimal sets constructed by Wang-Wang [WW77]. In Section 3 we introduce the notion of a saturated set. In Sections 4 and 5 we define the excess of a set and give an explicit formula for the excess of a box (Theorem 5.13). In Section 6 we show in Proposition 6.1 that all saturated minimal sets are boxes. Combined with our formula for the excess of box, we prove the first part of Theorem A, thus characterizing all minimal sets in terms of their enclosing boxes. In Section 7 we introduce cones and show that up to congruence, there is a unique disconnected minimal set. We also prove the second part of Theorem A and show the neighborhood of a minimal set is minimal. In Section 8 we study the graph $\mathcal{G}$ and classify which sets are efficient, uniquely minimal, dead and mortal.

\section{Acknowledgements}

We are extremely grateful to the anonymous referee for the helpful comments, corrections and suggestions. In particular, Theorem 7.9 was proven only after it was suggested by the referee as a potential application. We are thankful for helpful discussions with Joseph Briggs, who introduced us to Harper's Theorem. We also thank Nir Lazarovich for helpful comments and suggestions.

\section{Wang-Wang sets}

We recall the nested sequence of minimal sets in $X$ constructed by Wang-Wang.

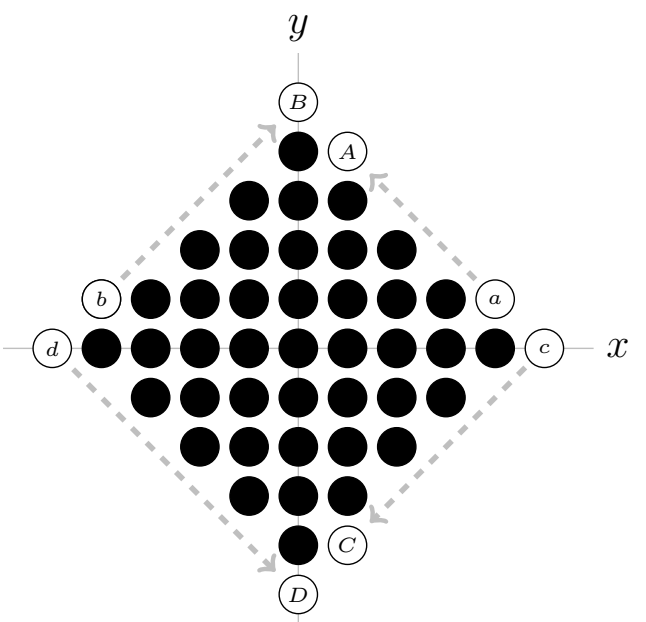

Figure 2.1: The black vertices denote the $\ell_{1}$-ball $W W_{r(n)}$ for some integer $n \geqslant 2$. The oriented lines show how one obtains the vertices $x_{r(n)+1}, \ldots, x_{r(n+1)}$.

Throughout this article, we fix the graph $X$ with vertex set $X^{0}=\mathbb{Z}^{2}$, where two vertices $(x, y),\left(x^{\prime}, y^{\prime}\right) \in X^{0}$ are joined by an edge if and only if $\left|x-x^{\prime}\right|+\left|y-y^{\prime}\right|=1$. 
Wang-Wang gave a nested sequence, $W W_{1} \subset W W_{2} \subset \ldots$, of minimal sets in $X$ such that $\left|W W_{n}\right|=n$ for all $n \geqslant 1$ [WW77]. Throughout this article, a Wang-Wang set is a subset $A \subset X^{0}$ that is congruent to $W W_{n}$ for some $n$. In the upcoming sections, we utilize them to prove our characterization of minimal sets in $X$.

In order to define the sets $\left(W W_{i}\right)$, it is enough to define a sequence of vertices $\left(x_{i}\right)$ for $1 \leqslant i \leqslant n$ such that $W W_{n}=\left\{x_{1}, \ldots, x_{n}\right\}$. The first five vertices in this sequence are given by coordinates:

$$
x_{1}=(0,0), x_{2}=(1,0), x_{3}=(0,1), x_{4}=(-1,0), x_{5}=(0,-1)
$$

Note that $\left\{x_{1}, \ldots, x_{5}\right\}$ is the $\ell_{1}$-ball in $X$ of radius 1 centered at $x_{1}$. Let $r(n):=2 n^{2}+2 n+1$ denote the size of an $\ell_{1}$-ball in $X$ of radius $n$. Suppose that the vertices $x_{1}, \ldots, x_{r(n)}$ have already been defined and that $W W_{r(n)}=\left\{x_{1}, \ldots, x_{r(n)}\right\}$ is the $\ell_{1}$-ball of radius $n$ centered at $x_{1}$, i.e., $W W_{r(n)}=\{x \in X|| x \mid \leqslant n\}$ (where $|$.$| is the \ell_{1}$-norm).

We use Figure 2.1 to define the vertices $x_{r(n)+1}, \ldots, x_{r(n+1)}$. We first set $x_{r(n)+1}$ to be the specific vertex adjacent to $W W_{r(n)}$ shown as vertex $a$ in Figure 2.1. The vertices $x_{r(n)+1}, \ldots, x_{r(n)+n}$ are those along the oriented line segment $\overrightarrow{a A}$ ordered by the given orientation. The next set of vertices are those along the segment $\overrightarrow{b B}$, then those on $\overrightarrow{c C}$, and finally those on $\overrightarrow{d D}$ (where each such sequence is again ordered by the given orientation).

The following lemma follows immediately from Wang-Wang's result:

Lemma 2.1. Let $A, B \subset X^{0}$. If $A$ is minimal and $|A| \leqslant|B|$, then $|\partial A| \leqslant|\partial B|$. If in addition $|\partial A|=|\partial B|$, then $B$ is minimal.

Proof. For every $m \geqslant 2$, it is easy to verify that either $\left|\partial W W_{m+1}\right|=\left|\partial W W_{m}\right|$ or $\left|\partial W W_{m+1}\right|=\left|\partial W W_{m}\right|+1$. It follows that $\left|\partial W W_{n}\right| \leqslant\left|\partial W W_{m}\right|$ if $n \leqslant m$. As every Wang-Wang set is minimal, $\left|\partial W W_{|A|}\right|=|\partial A|$ and $\left|\partial W W_{|B|}\right| \leqslant|\partial B|$; thus $|\partial A| \leqslant|\partial B|$. Now suppose that $|\partial A|=|\partial B|$. If for some $C \subset X^{0}$ we have $|B|=|C|$, then as $|A| \leqslant|C|$, we have $|\partial B|=|\partial A| \leqslant|\partial C|$. Thus $B$ is minimal.

\section{Saturated sets}

We define the notion of a saturated set, a subset of $X^{0}$ with the property that if any additional vertex is added to this set, then its boundary must increase.

Definition 3.1. A set $A \subset X^{0}$ is saturated if $|\partial(A \cup\{v\})|>|\partial A|$ for all $v \in X^{0} \backslash A$.

A configuration is a subset $(F, N) \subset X^{0} \times X^{0}$ such that $F \cap N=\emptyset$. We say that two configurations $(F, N)$ and $\left(F^{\prime}, N^{\prime}\right)$ are congruent if there is an automorphism $\phi \in \operatorname{Aut}(X)$ such that $\left(F^{\prime}, N^{\prime}\right)=(\phi(F), \phi(N))$. A set $A \subset X^{0}$ contains the configuration $(F, N)$ if $F \subset A$ and $N \cap A=\emptyset$. Some configurations are shown in Figure 3.1.

Lemma 3.2. If $A \subset X^{0}$ is saturated, then $A$ does not contain a configuration congruent to one shown in Figure 3.1. 


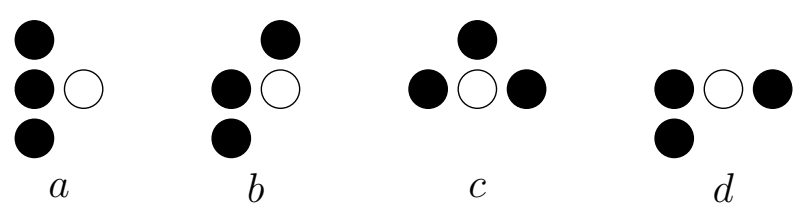

Figure 3.1: Four configurations $(F, N)$ are shown, where elements of $F$ are shown as black vertices and elements of $N$ are shown as white vertices. Lemma 3.2 ensures that a saturated set cannot contain a configuration congruent to one of the ones above.

Proof. Suppose $A$ contains a configuration $(F, N)$ from Figure 3.1, and let $v \in N$. By definition $v \notin A$. Furthermore, it is straightforward to check that $\partial(A \cup\{v\}) \leqslant \partial A$, contradicting our assumption that $A$ is saturated.

\section{Excess}

We introduce excess, a number associated to a subset $A \subset X^{0}$ that we will use in later sections to characterize minimal sets and to study the structure of the graph of minimal sets.

Definition 4.1. The excess of $A \subset X^{0}$ is defined to be

$$
\operatorname{Ex}(A):=\max \left\{|A|-|B| \mid B \subset X^{0} \text { is minimal and }|\partial A|=|\partial B|\right\} .
$$

The following lemma shows that the excess of $A$ is well-defined.

Lemma 4.2. For any finite $A \subset X^{0}$, there exists a minimal set $B \subset X^{0}$ such that $|\partial A|=|\partial B|$.

Proof. We first claim that if $n \geqslant 6$, then there exists a minimal set $B$ with $|\partial B|=n$. Indeed, as noted in the proof of Lemma 2.1, for every $m \geqslant 2$ either $\left|\partial W W_{m+1}\right|=\left|\partial W W_{m}\right|$ or $\left|\partial W W_{m+1}\right|=\left|\partial W W_{m}\right|+1$. Moreover, $\left\{\left|\partial W W_{m}\right| \mid m \in \mathbb{N}\right\}$ is unbounded. Since $\left|\partial W W_{2}\right|=6$, the claim follows.

If $|A|=1$, then $A$ is minimal. Otherwise, $|A| \geqslant 2=\left|W W_{2}\right|$, so Lemma 2.1 ensures that $|\partial A| \geqslant\left|W W_{2}\right|=6$. By the preceding claim, there exists a minimal set $B$ with $|\partial A|=|\partial B|$.

The next two lemmas follow almost immediately from the definition of excess.

Lemma 4.3. A finite set $A \subset X^{0}$ is minimal if and only if $\operatorname{Ex}(A) \geqslant 0$.

Proof. If $A$ is minimal, then by taking $B=A$ in the definition of excess, we get that $\operatorname{Ex}(A) \geqslant 0$. For the converse, if $\operatorname{Ex}(A) \geqslant 0$, then there exists a minimal set $B$ such that $|\partial A|=|\partial B|$ and $|A|-|B| \geqslant 0$. Thus, $A$ is minimal by Lemma 2.1.

Lemma 4.4. If $A, A^{\prime} \subset X^{0}$ are finite and $|\partial A|=\left|\partial A^{\prime}\right|$, then $\operatorname{Ex}(A)-\operatorname{Ex}\left(A^{\prime}\right)=|A|-\left|A^{\prime}\right|$. 
Proof. Since $|\partial A|=\left|\partial A^{\prime}\right|$, there exists a minimal set $B \subset X^{0}$ such that $\operatorname{Ex}(A)=|A|-|B|$ and $\operatorname{Ex}\left(A^{\prime}\right)=\left|A^{\prime}\right|-|B|$. Thus,

$$
\operatorname{Ex}(A)-\operatorname{Ex}\left(A^{\prime}\right)=(|A|-|B|)-\left(\left|A^{\prime}\right|-|B|\right)=|A|-\left|A^{\prime}\right| .
$$

\section{Boxes}

In this section, we define boxes. These are sets that are bounded by lines of slope 1 and -1. We prove some key facts regarding these sets and give an explicit formula for their excess. As a consequence, we determine which boxes are minimal sets.

Proposition 5.6 demonstrates that the following definition of a box is equivalent to the one given in the introduction.

Definition 5.1. A box in $X$ is a nonempty subset of $X^{0}$ of the form

$$
B(a, b, c, d):=\left\{(x, y) \in X^{0} \mid a \leqslant y-x \leqslant b \text { and } c \leqslant y+x \leqslant d\right\}
$$

for some $a, b, c, d \in \mathbb{Z}$.

Convention 5.2. There is an ambiguity when giving coordinates for boxes, which only arises for degenerate boxes of the form $B(a, b, c, d)$ where either $a=b$ or $c=d$. For example, $B(0,0,0,3)$ and $B(0,0,0,2)$ are the same box. To remedy this issue and ensure boxes can be uniquely parametrized, given a box $B(a, b, c, d)$ we implicitly assume that a and $c$ are maximal and that $b$ and $d$ are minimal out of all possible choices.

Given a subset $A \subset X^{0}$, recall that $N(A):=A \sqcup \partial A$. We next show that boxes are saturated. In the next section, we prove a converse to this statement for minimal sets (see Proposition 6.1).

Lemma 5.3. Every box is saturated.

Proof. Let $B=B(a, b, c, d)$ be a box. Suppose $v=(x, y) \in X^{0} \backslash B$. As $v \notin B, x$ and $y$ do not satisfy one of the four defining equations of $B$. Without loss of generality, we assume $x+y>d$. It follows that $(x+1, y)$ and $(x, y+1)$ are adjacent to $v$ and not contained in $N(B)$. As $|N(B \cup\{v\})| \geqslant|N(B)|+2$, we have

$|\partial(B \cup\{v\})|=|N(B \cup\{v\})|-|B \cup\{v\}| \geqslant(|N(B)|+2)-(|B|+1)=|\partial B|+1>|\partial B|$,

demonstrating that $B$ is saturated.

An extremal line of a box $B(a, b, c, d)$ is the set of solutions in $\mathbb{Z}^{2}$ to one of the four equations $y-x=a, y-x=b, y+x=c$ or $y+x=d$. By the above convention, a box intersects each of its extremal lines in at least one point. A corner of the box $B$ is an element of $B$ that lies on the intersection of two distinct extremal lines. A box has either zero, two, or four corners. An example of a box with two corners is shown in the left of Figure 5.1 and one with no corners is shown on the right of that figure. 


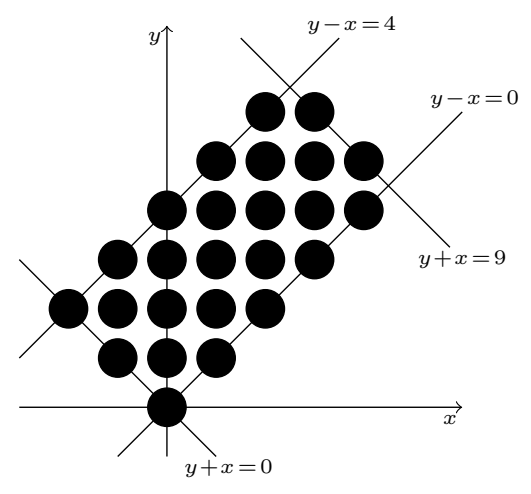

(a) The box $B(0,4,0,9)=B(4,9)$.

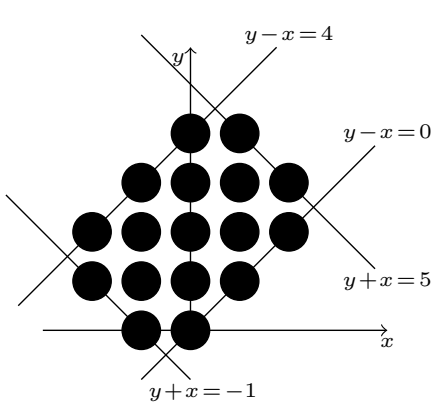

(b) The box $B(0,4,-1,5)=\hat{B}(4,6)$.

Figure 5.1

Definition 5.4. The modulus of a box $B(a, b, c, d)$ is the unordered pair $\{b-a, d-c\}$.

When Convention 5.2 is followed, it is evident that the modulus of a box is well-defined and is invariant under congruence. We intuitively expect that a box of modulus $\{\alpha, \beta\}$ is minimal when $|\alpha-\beta|$ is small. We precisely quantify how small $|\alpha-\beta|$ must be in Theorem 5.13 and Remark 5.14.

We now show that a box of modulus $\{\alpha, \beta\}$ is congruent to a "standard box" $B(\alpha, \beta)$ or $\hat{B}(\alpha, \beta)$ as defined below.

Notation 5.5. Define

$$
B(\alpha, \beta):=B(0, \alpha, 0, \beta)
$$

for any $\alpha, \beta \in \mathbb{N}$, and

$$
\hat{B}(\alpha, \beta):=B(0, \alpha,-1, \beta-1)
$$

for any even $\alpha, \beta \in \mathbb{N}$ (see Figure 5.1 for examples).

Proposition 5.6. Let $B=B(a, b, c, d)$ be a box. If $B$ has no corners, then $b-a$ and $d-c$ are both even and $B$ is congruent to $\hat{B}(b-a, d-c)$. Otherwise, $B$ is congruent to $B(b-a, d-c)$.

Proof. Suppose first that $B$ contains no corners. Then the intersection of the line $y-x=a$ with the line $y+x=c$ does not have integer coordinates, so $a$ and $c$ have opposite parity. Similarly, we deduce that $a$ and $d$ have opposite parity and that $b$ and $c$ have opposite parity. Thus $b-a$ and $d-c$ must both be even. Let $u$ be the vertex of $B$ which lies on the the line $y=x+a$ and has minimal $y$-value of all such possible choices. We can apply a translation which sends $u$ to the origin $(0,0)$. The resulting box is then $B(0, b-a,-1, d-c-1)=\hat{B}(b-a, d-c)$ as desired.

On the other hand, suppose that $B$ contains a corner $v$. Then there exists an automorphism of $X$ sending $v$ to the origin that maps $B$ to the box $B(b-a, d-c)$.

The next two lemmas calculate the size of a box and its boundary. 
Lemma 5.7. Let $B$ be a box with modulus $\{\alpha, \beta\}$. Then $|\partial B|=\alpha+\beta+4$.

Proof. Let $B=B(a, b, c, d)$ be a box of modulus $\{\alpha=b-a, \beta=d-c\}$. We can assume without loss of generality that $\alpha \leqslant \beta$. First suppose that $\alpha=0$, in which case $B$ is congruent to $B(0, \beta)$. Note that Convention 5.2 implies $\beta$ must be even. Since $|\partial B(0,0)|=4$ and $|\partial B(0,2 n+2)|=|\partial B(0,2 n)|+2$ for every $n \in \mathbb{N}$, it follows by induction that $|\partial B(0, \beta)|=\beta+4$ for all even $\beta$.

We thus assume that $\alpha \geqslant 1$. If $\alpha=\beta=1$, then $B$ is congruent to $B(1,1)$ and the formula $|\partial B|=\alpha+\beta+4=6$ clearly holds. We thus also assume that $\beta \geqslant 2$ and proceed by induction on $\beta$. We assume that the lemma is true for all boxes of modulus $\left\{\alpha^{\prime}, \beta^{\prime}\right\}$, where $\max \left(\alpha^{\prime}, \beta^{\prime}\right)<\beta$. Let $L$ and $L^{+}$be the lines with equations $y=-x+d$ and $y=-x+d+1$ respectively. Let $V:=\partial(L \cap B) \cap L^{+}$and observe that $|V|=|L \cap B|+1$. Let $B^{\prime}=B(a, b, c, d-1)$. Since $d-c \geqslant 2$ and $b-a \geqslant 1$, the preceding parametrization of $B^{\prime}$ is consistent with Convention 5.2. Thus $B^{\prime}$ has modulus $\{\alpha, \beta-1\}$. We observe that $N(B)=N\left(B^{\prime}\right) \sqcup V$ and $B=B^{\prime} \sqcup(B \cap L)$. The claim now follows from the equation below, where the last equality uses our induction hypothesis.

$$
|\partial B|=|N(B)|-|B|=\left(\left|N\left(B^{\prime}\right)\right|+|V|\right)-\left(\left|B^{\prime}\right|+|L \cap B|\right)=\left|\partial B^{\prime}\right|+1=\alpha+\beta+4 .
$$

Lemma 5.8. Let $\alpha, \beta \in \mathbb{N}$, we have that

$$
|B(\alpha, \beta)|=\left\lfloor\frac{\alpha \beta+\alpha+\beta+2}{2}\right\rfloor .
$$

Moreover, if $\alpha$ and $\beta$ are both even, then

$$
|\hat{B}(\alpha, \beta)|=\frac{\alpha \beta+\alpha+\beta}{2} .
$$

Proof. Let $p: X^{0} \rightarrow \mathbb{Z}$ be the projection map given by $(x, y) \mapsto y-x$. Let $B$ be a box and let $I$ be the interval $p(B)$. Thus $|B|=\sum_{i \in I}\left|p^{-1}(i) \cap B\right|$. We break the proof into cases depending on the type of box $B$ and the parity of $\alpha$ and $\beta$.

We first analyze the case where $B=B(\alpha, \beta)$. Suppose $\beta$ is even. It follows that $\left|p^{-1}(i)\right|=\frac{\beta}{2}+1$ for even $i \in I$ and $\left|p^{-1}(i)\right|=\frac{\beta}{2}$ for odd $i \in I$. Thus, if $\alpha$ is even, then

$$
|B|=\left(\frac{\alpha}{2}+1\right)\left(\frac{\beta}{2}+1\right)+\left(\frac{\alpha}{2} \frac{\beta}{2}\right)=\frac{\alpha \beta+\alpha+\beta+2}{2}=\left\lfloor\frac{\alpha \beta+\alpha+\beta+2}{2}\right\rfloor .
$$

If $\alpha$ is odd, we have:

$$
|B|=\left(\frac{\alpha+1}{2}\right)\left(\frac{\beta}{2}+1\right)+\left(\frac{\alpha+1}{2}\right)\left(\frac{\beta}{2}\right)=\frac{\alpha \beta+\alpha+\beta+1}{2}=\left\lfloor\frac{\alpha \beta+\alpha+\beta+2}{2}\right\rfloor .
$$

The last equality follows since $\frac{\alpha \beta+\alpha+\beta+1}{2}$ is equal to $|B|$ and hence it is an integer.

On the other hand, suppose that $\beta$ is odd. In this case, it follows that $\left|p^{-1}(i)\right|=\frac{\beta+1}{2}$ for all $i \in I$. Thus,

$$
|B|=\sum_{j=0}^{\alpha} \frac{\beta+1}{2}=(\alpha+1)\left(\frac{\beta+1}{2}\right)=\frac{\alpha \beta+\alpha+\beta+1}{2}=\left\lfloor\frac{\alpha \beta+\alpha+\beta+2}{2}\right\rfloor .
$$


Finally, let $B=\hat{B}(\alpha, \beta)$ where both $\alpha$ and $\beta$ are even. It follows that $\left|p^{-1}(i)\right|=\frac{\beta}{2}$ for even $i \in I$ and $\left|p^{-1}(i)\right|=\frac{\beta}{2}+1$ for odd $i \in I$. Thus,

$$
|B|=\left(\frac{\alpha}{2}+1\right) \frac{\beta}{2}+\frac{\alpha}{2}\left(\frac{\beta}{2}+1\right)=\frac{\alpha \beta+\alpha+\beta}{2} .
$$

Remark 5.9. If $\alpha, \beta \in \mathbb{N}$ are both even, then by Lemma 5.7 and Lemma 5.8 we have $|\partial \hat{B}(\alpha, \beta)|=|\partial B(\alpha, \beta)|$ and $|\hat{B}(\alpha, \beta)|+1=|B(\alpha, \beta)|$.

A standard line is a set of the form $\{(x, y) \mid y-x=w\}$ or $\{(x, y) \mid y+x=w\}$ for some $w \in \mathbb{Z}$. The following lemma allows us to take a nested sequence of subsets of a box, all with the same size boundary. This lemma will be used in Sections 7 and 8.3 as well as here.

Lemma 5.10. Let $B$ be a box of modulus $\{\alpha, \beta\}$, and let $L$ be an extremal line of $B$. Set $n=|L \cap B|-1$. Then the following are true:

1. If $\alpha, \beta \geqslant 2$, then there exist sets $B=B_{0} \supset B_{1} \supset \ldots \supset B_{n}$ such that $\left|\partial B_{i}\right|=\left|\partial B_{i-1}\right|$ for all $1 \leqslant i \leqslant n$.

2. $\operatorname{Ex}(B) \leqslant n$

3. If $L^{\prime}$ is a standard line that intersects $B$, then $\operatorname{Ex}(B) \leqslant\left|L^{\prime} \cap B\right|$.

Proof. Without loss of generality, we may assume that $B=B(a, b, c, d)$ and that $L$ is the line with equation $y=-x+d$.

We first suppose that $\alpha, \beta \geqslant 2$, and we prove claims (1) and (2) in this case. Let $v_{1}=\left(x_{1}, y_{1}\right), \ldots, v_{n+1}=\left(x_{n+1}, y_{n+1}\right)$ be the vertices of $L \cap B$, ordered so that $x_{1}<x_{2}<$ $\ldots<x_{n+1}$. Let $B_{i}:=B \backslash\left\{v_{1}, \ldots, v_{i}\right\}$. It follows from our hypothesis on the modulus of $B$ that, for each $1 \leqslant i \leqslant n$, the vertices $\left(x_{i}, y_{i}\right),\left(x_{i}-1, y_{i}\right),\left(x_{i}+1, y_{i}\right)$ and $\left(x_{i}, y_{i}-1\right)$ are each contained in $N\left(B_{i}\right)$ and $\left(x_{i}, y_{i}+1\right)$ is not. Thus $N\left(B_{i-1}\right)=N\left(B_{i}\right) \sqcup\left\{\left(x_{i}, y_{i}+1\right)\right\}$ for each $1 \leqslant i \leqslant n$, and so $\left|\partial B_{i}\right|=\left|\partial B_{i-1}\right|$. This shows (1). To see (2), note that $B_{n+1}$ is a box whose modulus is either $\{\alpha-1, \beta\}$ or $\{\alpha, \beta-1\}$. By Lemma 5.7, $\left|\partial B_{n+1}\right|=|\partial B|-1$. Thus, any minimal set of size $\left|B_{n+1}\right|=|B|-(n+1)$ must have boundary of size at most $|\partial B|-1$. It follows that $\operatorname{Ex}(B) \leqslant n$. Thus, (2) follows in this case.

We now suppose that $\beta:=d-c \geqslant \alpha:=b-a$ and that $\alpha \leqslant 1$, and we prove (2) for this remaining case. As before, let $L$ be the line with equation $y=-x+d$. Since $\alpha \leqslant 1, L$ intersects $B$ in a single vertex $v$. It follows that $B^{\prime}:=B \backslash v$ is a box and is of strictly smaller modulus. Thus Lemma 5.7 implies that $\left|\partial B^{\prime}\right|<|\partial B|$. It follows from Lemma 2.1 that any minimal set of size at most $|B|-1$ has boundary of size at most $|\partial B|-1$. This implies $\operatorname{Ex}(B) \leqslant 0$ as required. If $Q$ is any other extremal line of $B$, then $|Q \cap B| \geqslant|L \cap B|=1$ and we also get that $\operatorname{Ex}(B) \leqslant|Q \cap B|-1$. Thus, (2) follows.

Finally, to see (3), suppose $L^{\prime}$ is a standard line that intersects $B$, and let $L^{\prime \prime}$ be the extremal line of $B$ that is parallel to $L^{\prime}$. It follows that $\left|L^{\prime \prime} \cap B\right| \leqslant\left|L^{\prime} \cap B\right|+1$. By what we have shown, we get that $\operatorname{Ex}(B) \leqslant\left|L^{\prime \prime} \cap B\right|-1$ as required. 
Remark 5.11. By considering the box $B=B(2,2)$, which has excess one, we see that the bounds for $\operatorname{Ex}(B)$ given in the previous lemma are sharp. See also Lemma 5.12 for more boxes where the bound is achieved.

To calculate the excess of an arbitrary box, we first compute the excess of a box with modulus $\{\alpha, \beta\}$, where $|\alpha-\beta| \leqslant 1$.

Lemma 5.12. For every $n \in \mathbb{N}$,

- $\operatorname{Ex}(B(2 n, 2 n))=n$;

- $\operatorname{Ex}(B(2 n+2,2 n+3))=n$;

- $\operatorname{Ex}(B(2 n+1,2 n+1))=n$;

- $\operatorname{Ex}(B(2 n+1,2 n+2))=n$;

- $\operatorname{Ex}(\hat{B}(2 n, 2 n))=n-1$.

Proof. We first remark that if $A \subset X^{0}$ is minimal, then $|\partial A|=\left|\partial W W_{|A|}\right|$. Thus for any minimal set $A$ of $X, \operatorname{Ex}(A)=\max \left\{k|| \partial W W_{|A|-k}|=| \partial W W_{|A|} \mid\right\}$.

Let $Y$ be one of $B(2 n, 2 n), B(2 n+2,2 n+3), B(2 n+1,2 n+1)$ or $B(2 n+1,2 n+2)$. Then $Y$ is congruent to a Wang-Wang set $W W_{m}$ for some $m$. It can be verified by Lemma 5.10(1) that $\left|\partial W W_{m-i}\right|=\left|\partial W W_{m}\right|$ if and only if $i \leqslant n$. This gives the required formula for the excess of $Y$. Finally, by Lemma 4.4 and Remark 5.9, $\operatorname{Ex}(\hat{B}(2 n, 2 n))=$ $\operatorname{Ex}(B(2 n, 2 n))-1=n-1$.

We are now ready to calculate the excess of any box.

Theorem 5.13. Suppose $\alpha, \beta \in \mathbb{N}$. Let $r:=\frac{\alpha+\beta}{2}, k:=\frac{|\beta-\alpha|}{2}$. Then

$$
\operatorname{Ex}(B(\alpha, \beta))=\left\lfloor\frac{\lfloor r\rfloor-k^{2}}{2}\right\rfloor .
$$

Moreover, when $\alpha, \beta \in \mathbb{N}$ are even, then

$$
\operatorname{Ex}(\hat{B}(\alpha, \beta))=\frac{r-k^{2}-2}{2} .
$$

Proof. By applying an automorphism of $X$, we may assume that $\beta \geqslant \alpha$. Note that $\alpha=r-k$ and $\beta=r+k$. We break the argument into two cases.

Case $A: \alpha$ and $\beta$ have the same parity.

In this case, $r$ and $k$ are both integers. Lemma 5.8 yields the equations:

$$
\begin{gathered}
|B(r, r)|=\left\lfloor\frac{r^{2}+2 r+2}{2}\right\rfloor=\left\lfloor\frac{r^{2}}{2}\right\rfloor+r+1, \\
|B(r-k, r+k)|=\left\lfloor\frac{r^{2}-k^{2}+2 r+2}{2}\right\rfloor=\left\lfloor\frac{r^{2}-k^{2}}{2}\right\rfloor+r+1 .
\end{gathered}
$$


Setting $r=2 m+\epsilon$ where $\epsilon \in\{0,1\}$ and $m \in \mathbb{Z}$, we get the equation:

$$
\left\lfloor\frac{r^{2}-k^{2}}{2}\right\rfloor-\left\lfloor\frac{r^{2}}{2}\right\rfloor=\left\lfloor\frac{\epsilon^{2}-k^{2}}{2}+2 m^{2}+2 m \epsilon\right\rfloor-\left\lfloor\frac{\epsilon^{2}}{2}+2 m^{2}+2 m \epsilon\right\rfloor=\left\lfloor\frac{\epsilon-k^{2}}{2}\right\rfloor
$$

It follows from Lemma 5.7 that $|\partial B(r, r)|=|\partial B(r-k, r+k)|$. Lemma 4.4 then implies

$$
\begin{aligned}
\operatorname{Ex}(B(\alpha, \beta)) & =\operatorname{Ex}(B(r-k, r+k))=\operatorname{Ex}(B(r, r))-|B(r, r)|+|B(r-k, r+k)| \\
& =\operatorname{Ex}(B(r, r))+\left\lfloor\frac{r^{2}-k^{2}}{2}\right\rfloor-\left\lfloor\frac{r^{2}}{2}\right\rfloor=\left\lfloor\operatorname{Ex}(B(r, r))+\frac{\epsilon-k^{2}}{2}\right\rfloor .
\end{aligned}
$$

When $r$ is even (and so $\epsilon=0), \operatorname{Ex}(B(r, r))=\frac{r}{2}$ by Lemma 5.12. Substituting this into the above equation yields

$$
\operatorname{Ex}(B(\alpha, \beta))=\left\lfloor\frac{r-k^{2}}{2}\right\rfloor=\left\lfloor\frac{\lfloor r\rfloor-k^{2}}{2}\right\rfloor .
$$

When $r$ is odd (and so $\epsilon=1), \operatorname{Ex}(B(r, r))=\frac{r-1}{2}$ by Lemma 5.12. Thus,

$$
\operatorname{Ex}(B(\alpha, \beta))=\left\lfloor\frac{r-1}{2}+\frac{1-k^{2}}{2}\right\rfloor=\left\lfloor\frac{\lfloor r\rfloor-k^{2}}{2}\right\rfloor .
$$

Case B: $\alpha$ and $\beta$ have different parity. We can write $r=s+\frac{1}{2}$ and $k=t+\frac{1}{2}$, for some $s, t \in \mathbb{Z}$. Note that $\lfloor r\rfloor=s$, so we need to show

$$
\operatorname{Ex}(B(\alpha, \beta))=\left\lfloor\frac{s-t^{2}-t-\frac{1}{4}}{2}\right\rfloor=\left\lfloor\frac{s}{2}-\frac{1}{8}\right\rfloor-\frac{t^{2}+t}{2}
$$

The last equality follows since $t^{2}+t$ is even. Lemma 5.8 now yields

$$
\begin{aligned}
|B(s, s+1)| & =\left\lfloor\frac{s^{2}+3 s+3}{2}\right\rfloor \\
|B(\alpha, \beta)|=|B(s-t, s+t+1)| & =\left\lfloor\frac{s^{2}+3 s+3-t^{2}-t}{2}\right\rfloor=|B(s, s+1)|-\frac{t^{2}+t}{2},
\end{aligned}
$$

where the last equality follows again because $t^{2}+t$ is even.

As in Case A,

$$
\begin{aligned}
\operatorname{Ex}(B(s-t, s+t+1)) & =\operatorname{Ex}(B(s, s+1))-|B(s, s+1)|+|B(s-t, s+t+1)| \\
& =\operatorname{Ex}(B(s, s+1))-\frac{t^{2}+t}{2}
\end{aligned}
$$

So by Equation 5.1, we need only to verify that $\operatorname{Ex}(B(s, s+1))=\left\lfloor\frac{s}{2}-\frac{1}{8}\right\rfloor$, or equivalently:

$$
\left\lfloor\frac{s}{2}-\frac{1}{8}-\operatorname{Ex}(B(s, s+1))\right\rfloor=0 .
$$


Lemma 5.12 ensures $\operatorname{Ex}(B(s, s+1))=\frac{s}{2}-1$ when $s$ is even, and $\operatorname{Ex}(B(s, s+1))=\frac{s}{2}-\frac{1}{2}$ when $s$ is odd, so the preceding equation is satisfied in both cases.

Finally, Lemma 4.4 and Remark 5.9 imply that $\operatorname{Ex}(\hat{B}(\alpha, \beta))=\operatorname{Ex}(B(\alpha, \beta))-1=$ $\frac{r-k^{2}-2}{2}$ for even $\alpha, \beta \in \mathbb{N}$.

Remark 5.14. By combining Lemma 4.3 and Theorem 5.13, we have a complete characterization of which boxes are minimal.

\section{Characterizing minimal sets}

In this section we prove Theorem 6.4, giving our first characterization of minimal sets in the graph $X=\mathbb{Z}_{\ell_{1}}^{2}$. We also prove Proposition 6.8 , which characterizes boxes as precisely the sets that are both saturated and $\ell_{\infty}$-connected.

We first explain how to deduce Theorem 6.4 from the following proposition, whose proof occupies the remainder of this section.

Proposition 6.1. If $A \subset X$ is minimal and saturated, then it is a box.

Definition 6.2. Given a finite set $A \subset X^{0}$, the enclosing box, denoted enc $(A)$, is the smallest box that contains $A$.

The enclosing box of a set is well-defined, as the intersection of boxes is itself a box. The enclosing box of a minimal set is the unique smallest saturated set containing it:

Lemma 6.3. Let $A \subset X^{0}$ be a minimal set, and let $A=A_{0} \subset A_{1} \subset A_{2} \subset \ldots$ be a (possibly finite) maximal sequence of nested minimal sets such that $\left|A_{i+1}\right|=\left|A_{i}\right|+1$ for each $i$. Then $A_{N}=\operatorname{enc}(A)$ for some $N \geqslant 0$ and $|\partial A|=|\partial(\operatorname{enc}(A))|$.

Proof. Let $N \geqslant 0$ be the largest integer such that $\left|\partial A_{N}\right|=|\partial A|$. Such an integer exists as there are minimal sets with arbitrarily large boundaries (see the proof of Lemma 4.2 for instance). To prove the lemma, it suffices to show that $A_{N}=\operatorname{enc}(A)$.

We first claim that $A_{N}$ must be saturated. For a contradiction, suppose otherwise. It follows there exists a set $A_{N}^{\prime} \supset A_{N}$ (not necessarily equal to $A_{N+1}$ ) such that $\left|\partial A_{N}^{\prime}\right|=\left|\partial A_{N}\right|$ and $\left|A_{N}^{\prime}\right|=\left|A_{N}\right|+1$. By Lemma 2.1, $A_{N}^{\prime}$ is minimal. The maximality of our nested sequence, ensures it contains a set $A_{N+1}$. As $A_{N+1}$ is minimal and $\left|A_{N+1}\right|=\left|A_{N}^{\prime}\right|$, it follows that $\left|\partial A_{N+1}\right|=\left|\partial A_{N}\right|$, contradicting our choice of $N$.

As $A_{N}$ is saturated and minimal, it is a box by Proposition 6.1. If $\operatorname{enc}(A) \neq A_{N}$, then enc $(A)$ must be a proper subset of $A_{N}$. However, in this case, we then have that $|\partial(\operatorname{enc}(A))|<\left|\partial A_{N}\right|$ by Lemma 5.7, contradicting our choice of $N$. Thus $A_{N}=\operatorname{enc}(A)$, and the lemma follows.

We are ready to prove our first characterization of minimal sets, using Proposition 6.1.

Theorem 6.4. Let $A \subset X^{0}, N:=|\operatorname{enc}(A) \backslash A|$ and $E=\operatorname{Ex}(\operatorname{enc}(A))$. Then $A$ is minimal if and only if $|\partial A|=|\partial(\operatorname{enc}(A))|$ and $E \geqslant N$. 
Proof. First suppose $A$ is minimal. By Lemma $6.3,|\partial A|=|\partial \operatorname{enc}(A)|$. Thus by Definition 4.1, $E \geqslant N$. Conversely, suppose that $|\partial A|=|\partial(\operatorname{enc}(A))|$ and $E \geqslant N$. It follows from Definition 4.1 that there exists a minimal set $C$ such that $|\partial C|=|\partial(\operatorname{enc}(A))|=|\partial A|$ and

$$
|C|=|\operatorname{enc}(A)|-E \leqslant|\operatorname{enc}(A)|-N=|A| \text {. }
$$

By Lemma 2.1, $A$ is minimal.

We now begin our proof of Proposition 6.1. We first establish some terminology regarding the $\ell_{\infty}$-metric on $X^{0}$. We recall the $\ell_{\infty}$-metric (also known as the Chebyshev, maximum, or chessboard metric) on $X^{0}$, which is defined by

$$
d_{\infty}\left((x, y),\left(x^{\prime}, y^{\prime}\right)\right):=\max \left(\left|x-x^{\prime}\right|,\left|y-y^{\prime}\right|\right) .
$$

Two vertices $u, v \in X^{0}$ are said to be $\ell_{\infty}$-adjacent if $d_{\infty}(u, v)=1$. An $\ell_{\infty}$-path is a sequence $\left(u_{i}\right)_{i=0}^{n}$ of elements of $X^{0}$ such that $u_{i-1}$ and $u_{i}$ are $\ell_{\infty}$-adjacent for every $0<i \leqslant n$. A subset $A \subset X^{0}$ is $\ell_{\infty}$-connected if any pair of vertices in $A$ are the endpoints of an $\ell_{\infty}$-path contained in $A$. An $\ell_{\infty}$-component of $A$ is a maximal $\ell_{\infty}$-connected subset of $A$.

Remark 6.5. When we use the terms path, connected and adjacent without the prefix $\ell_{\infty}$, it is assumed we are referring to these properties in the graph $X$ or equivalently, in $X^{(0)}$ equipped with $\ell_{1}$-metric.

The next two lemmas will be needed to prove Proposition 6.8, our characterization of boxes.

Lemma 6.6. Suppose $A \subset X^{0}$ is saturated and $C \subseteq A$ is an $\ell_{\infty}$-component of $A$ that is not contained in a standard line. Then $C$ contains a pair of adjacent vertices.

Proof. As $C$ is $\ell_{\infty}$-connected and not contained in a standard line, it contains an $\ell_{\infty}$-path $\gamma=\left(u_{0}, \ldots u_{n}\right)$ such that $u_{0}$ and $u_{n}$ do not lie on the same standard line. We assume no $u_{i}$ is adjacent to $u_{i+1}$, otherwise we are done. Thus there exists an $i$ such that $u_{i-1}, u_{i}$ and $u_{i+1}$ do not lie in the same standard line. By applying an automorphism of $X$, we may assume $u_{i-1}=(1,-1), u_{i}=(0,0)$ and $u_{i+1}=(1,1)$. As $A \supseteq C$ is saturated, Lemma 3.2 ensures that $(1,0)$ is contained in $A$. As $C$ is an $\ell_{\infty}$-component of $A,(1,0) \in C$. We are done as $(1,0)$ is adjacent to $u_{i}$.

Lemma 6.7. If $A \subset X^{0}$ is a finite saturated set, then every $\ell_{\infty}$-component of $A$ is a box.

Proof. Let $C$ be an $\ell_{\infty}$-component of $A$. If $C$ is contained in a standard line, then we are done. Otherwise, by Lemma 6.6, $C$ contains two adjacent vertices. In particular, $C$ contains a box that is not contained in a standard line. Thus, up to congruence, $C$ contains a box $B=B(a, b, c, d)$ that is not contained in a standard line and is maximal, i.e. is not contained in any other box contained in $C$. We will show that $B=C$.

Assume for a contradiction that $B$ is a proper subset of $C$. As $C$ is $\ell_{\infty}$-connected, there exists an $\ell_{\infty}$-path from $B$ to $C \backslash B$ which is contained in $C$. Thus, there are vertices $w=(x, y) \in B$ and $v=\left(x^{\prime}, y^{\prime}\right) \in C \backslash B$ which are $\ell_{\infty}$-adjacent. By symmetry, we may assume that $x^{\prime}+y^{\prime}>d$. 
We claim that we may assume that $x+y=d$. For suppose that $x+y \neq d$, then as $w$ is $\ell_{\infty}$ adjacent to $v$, we have $x+y=d-1$ and $v=(x+1, y+1)$. At least one of $(x, y+1)$ or $(x+1, y)$ is contained in $B$, since $B$ is not contained in a standard line. By replacing $w$ with such a vertex, we may assume $x+y=d$.

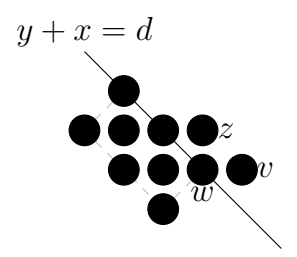

Figure 6.1

We now show that the box $B^{\prime}:=B(a, b, c, d+1)$ is contained in $C$, contradicting the maximality of $B$. We first show that $C$ contains a vertex of $B^{\prime} \backslash B$. If $v \in B^{\prime}$, then we are done. Otherwise, either $x^{\prime}+y^{\prime}=d+2$, or $y^{\prime}-x^{\prime}<a$, or $y^{\prime}-x^{\prime}>b$. If $y^{\prime}-x^{\prime}<a$ and $x^{\prime}+y^{\prime} \neq d+2$ - as is shown in Figure 6.1 - then $v=(x+1, y)$ and $y-x=a$. As $B$ is not contained in a standard line, $(x-1, y+1) \in A$. Thus by Lemma 3.2, $z:=(x, y+1) \in A$. Since $C$ is an $\ell_{\infty}$-component of $A$, we have that $z \in C \cap\left(B^{\prime} \backslash B\right)$. If $y^{\prime}-x^{\prime}>b$ and $x^{\prime}+y^{\prime} \neq d+2$, then a similar argument demonstrates that $B^{\prime} \backslash B$ contains a vertex of $C$. Finally, if $x^{\prime}+y^{\prime}=d+2$, then $v=(x+1, y+1)$. As $B$ is not contained in a standard line, either $(x+1, y-1) \in B$ or $(x-1, y+1) \in B$. By Lemma 3.2, $(x+1, y) \in A$ in the first case and $(x, y+1) \in A$ in the second. In either case, using the fact that $C$ is an $\ell_{\infty}$-component of $A$, we deduce there exists a vertex in $C \cap\left(B^{\prime} \backslash B\right)$.

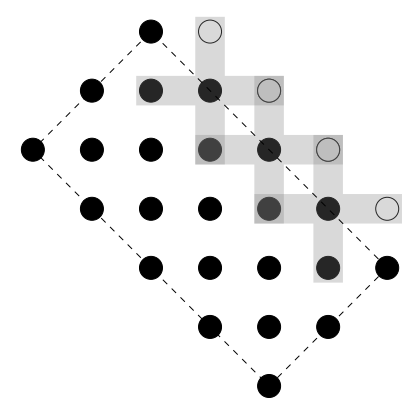

Figure 6.2: Black vertices are in $B$ and white vertices are in $B^{\prime} \backslash B$, where $B^{\prime}=B(0,8,0,5)$ and $B=B(0,8,0,4)$. The three overlapping Swiss crosses can be used to deduce, via Lemma 3.2, that if $C$ contains a single vertex of $B^{\prime} \backslash B$, then $B^{\prime} \subset C$.

By the previous paragraph, there exists a vertex $z=\left(x^{\prime \prime}, y^{\prime \prime}\right) \in C \cap\left(B^{\prime} \backslash B\right)$. Note that $\left(x^{\prime \prime}, y^{\prime \prime}-1\right) \in B$. If $\left(x^{\prime \prime}, y^{\prime \prime}-2\right) \in B$, then $\left(x^{\prime \prime}+1, y^{\prime \prime}-1\right) \in C$ by Lemma 3.2. Similarly, if $\left(x^{\prime \prime}-1, y^{\prime \prime}\right) \in B$, and if $\left(x^{\prime \prime}-2, y^{\prime \prime}\right) \in B$, then $\left(x^{\prime \prime}-1, y^{\prime \prime}+1\right) \in C$. By repeating these arguments, we see that $B^{\prime} \subset A$ (see Figure 6.2) as claimed, and we get a contradiction.

The following proposition characterizes $\ell_{\infty}$-connected, saturated subsets of $X^{0}$ as boxes.

Proposition 6.8. A finite subset $A \subset X^{0}$ is a box if and only if it is $\ell_{\infty}$-connected and saturated. 
Proof. If $A$ is a box, then it is finite, $\ell_{\infty}$-connected and saturated by Lemma 5.3. For the other direction, Lemma 6.7 implies that an $\ell_{\infty}$-connected saturated set is a box.

The next two lemmas, together with Proposition 6.8, show that a finite minimal saturated subset of $X^{0}$ is a box (Proposition 6.1).

Lemma 6.9. Let $A \subset X^{0}$ be a finite, saturated subset, and let $C_{1}$ and $C_{2}$ be distinct $\ell_{\infty}$-components of $A$ such that there exists a vertex $(x, y) \in \partial C_{1} \cap \partial C_{2}$. Then $C_{1}$ and $C_{2}$ are boxes, and, up to applying an automorphism of $X,(x-1, y)$ is a corner of $C_{1}$ and $(x+1, y)$ is a corner of $C_{2}$. Moreover, if $C$ is an $\ell_{\infty}$-component of $A$ with $(x, y) \in \partial C$, then $C$ is equal to either $C_{1}$ or $C_{2}$.

Proof. By Lemma 6.7, $C_{1}$ and $C_{2}$ are boxes. By possibly applying an automorphism of $X$, we assume that $(x-1, y) \in C_{1}$.

Note that $(x, y) \notin A$ and $(x, y \pm 1) \notin A$, for otherwise $C_{1}$ and $C_{2}$ would be joined by an $\ell_{\infty}$-path in $A$. Thus it must be the case that $(x+1, y) \in C_{2}$. In particular, the only vertices of $A$ adjacent to $(x, y)$ are $(x-1, y)$ and $(x+1, y)$. It follows that the only $\ell_{\infty}$-components of $A$ which contain $(x, y)$ in their boundary are precisely $C_{1}$ and $C_{2}$.

Moreover, $(x-1, y \pm 1) \notin A$ since $A$ cannot contain configuration $(d)$ in Figure 3.1 by Lemma 3.2. We deduce that $(x-1, y)$ and $(x+1, y)$ are corners of $C_{1}$ and $C_{2}$ respectively.

For the next lemma, we let $c(A)$ denote the number of $\ell_{\infty}$-components of a subset $A \subset X^{0}$.

Lemma 6.10. Let $A$ be a finite saturated subset of $X^{0}$. Then there exists a set $A^{\prime} \subset X^{0}$ such that $\left|A^{\prime}\right|=|A|$ and $\left|\partial A^{\prime}\right| \leqslant|\partial A|-(c(A)-1)$. In particular, if $A$ is minimal, then it is $\ell_{\infty}$-connected.

Proof. We first recall that every $\ell_{\infty}$-component of $A$ is a box by Lemma 6.7 . We prove the lemma by induction on $c(A)=n$. The base case $n=1$ trivially follows by setting $A^{\prime}=A$. When $n=2$, then $A^{\prime}$ is obtained from $A$ by translating one of the two $\ell_{\infty}$-components to reduce the boundary of the set by 1 (this is possible as these components are boxes). Now suppose $c(A)>2$ and that for all finite saturated sets $S$, with $c(S)<c(A)$ there exists a set $S^{\prime} \subset \mathcal{X}$ such that $\left|S^{\prime}\right|=|S|$ and $\left|\partial S^{\prime}\right| \leqslant|\partial S|-(c(S)-1)$.

Given a box $B=B(a, b, c, d)$ we say that the line $y=-x+d$ is the $N E$ extremal line of $B$ and that the line $y=-x+c$ is a $S W$ extremal line of $B$. Consider the smallest box containing $A$ and let $L$ be its NE extremal line. Let $C$ be an $\ell_{\infty}$-component of $A$ which contains a vertex of $L$. Since $A$ is saturated, so is $C$ and hence by Proposition 6.8 , the set $C$ is a box. Let $\bar{C}=A \backslash C$. By Lemma 6.9 , any vertex of $\partial C \cap \partial \bar{C}$ is adjacent to a corner of $C$ that does not lie on $L$. Thus, $|\partial C \cap \partial \bar{C}| \leqslant 2$, and we get that: $|\partial C|+|\partial \bar{C}| \leqslant|\partial A|+2$.

We will now show that we can replace $\bar{C}$ by a different set so that the union of this set with $C$ satisfies the claim. If $|\bar{C}|=1$, then $c(A)=2$ and we reduce to a base case. If $|\bar{C}|=2$, then set $W:=\{(0,0),(-1,1)\}$. Otherwise set $W=W W_{|\bar{C}|}$. Now since $A$ is saturated, $\bar{C}$ is also saturated and we have $c(\bar{C})=c(A)-1=n-1$. By the induction 


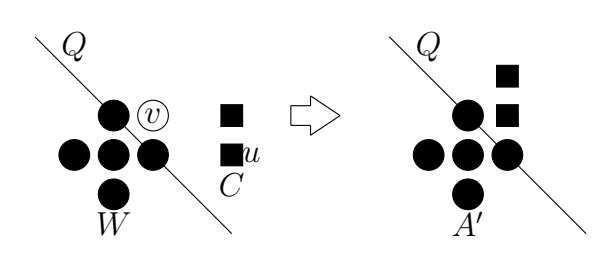

Figure 6.3: Arranging $W$ and $C$ to get $A^{\prime}$

hypothesis, there exists a set $P$ such that $|P|=|\bar{C}|$ and $|\partial P| \leqslant|\partial \bar{C}|-(n-2)$. As $W$ is minimal, $|\partial W| \leqslant|\partial P|$. Thus, $|\partial W| \leqslant|\partial \bar{C}|-(n-2)$.

We now translate $W$ and $C$ appropriately in order to define $A^{\prime}$. Let $Q$ be the line given by $y+x=0$. Since $W$ is a Wang-Wang set of size greater than two or is congruent to $\{(0,0),(-1,1)\}$, we can apply an automorphism so that $W \subset\{(x, y) \mid y+x \leqslant 0\}$ and $|W \cap Q| \geqslant 2$. Thus there exists some $v \in \partial W$ that is adjacent to two vertices of $W \cap Q$. Let $u \in C$ be a vertex on the SW extremal line of $C$. By translating $C$, we can suppose that $u$ and $v$ coincide. Setting $A^{\prime}:=W \cup C$, we get (see Figure 6.3)

$$
\left|\partial A^{\prime}\right| \leqslant|\partial C|+|\partial W|-3 .
$$

Thus

$$
\left|\partial A^{\prime}\right| \leqslant|\partial C|+|\partial W|-3 \leqslant|\partial C|+|\partial \bar{C}|-(n-2)-3 \leqslant|\partial A|-(n-1)
$$

and

$$
\left|A^{\prime}\right|=|W|+|C|=|\bar{C}|+|C|=|A| .
$$

This concludes the proof of the lemma.

Proof of Proposition 6.1. Suppose $A \subset X^{0}$ is minimal and saturated. By Lemma 6.10, $A$ is $\ell_{\infty}$-connected. Lemma 6.7 now implies that $A$ is a box.

\section{$7 \quad$ Minimal sets and cones}

In this section we prove another characterization of minimal sets in terms of cones and show that (almost) all minimal sets are connected. We also show the neighborhood of a minimal set is minimal.

\subsection{Minimal sets are connected}

Theorem 7.1. A minimal set in $X$ is connected if and only if it is not congruent to $B(0,2)$.

Before proving the above theorem, we need to first define cones and prove a series of lemmas. 
Definition 7.2. A cone is a subset of $X^{0}$ congruent to $C_{0}:=\{(x, y) \mid y-x \geqslant 0, y+x \geqslant 0\}$. An extremal ray of the cone $C_{0}$ is the intersection of $C_{0}$ with either the line $y=x$ or $y=-x$. An extremal ray of a cone is the image of an extremal ray of $C_{0}$ under the given congruence. A cone at the vertex $v \in X^{0}$ is a cone whose two extremal rays intersect at $v$. If $v \in X^{0}$, then the cone above $v$ is a cone at $v$ that is translation-equivalent to $C_{0}$; the cone to the right of $v$ is a cone at $v$ that is translation-equivalent to $C_{0}$ rotated clockwise $90^{\circ}$. The cones below $v$ and to the left of $v$ are defined analogously.

Remark 7.3. Given any box $B \subset X^{0}$, it readily follows that $X^{0} \backslash B$ is a finite union of cones.

Lemma 7.4. Let $A \subset X^{0}$ be a finite set such that $|\operatorname{enc}(A) \backslash A|<\operatorname{Ex}(\operatorname{enc}(A))$ and $X^{0} \backslash A$ is a union of cones. Suppose $v \in A$ and there is a cone $C$ based at $v$ such that $C \cap A=\{v\}$. Without loss of generality, we can suppose $v=(0,0)$ and that $C$ is the cone above $v$. Then $(-1,-1),(0,-1),(1,-1) \in A$.

Proof. Suppose $B=B(a, b, c, d):=\operatorname{enc}(A)$. Let $A^{\prime}:=A \backslash\{v\}$. Observe that $X^{0} \backslash A^{\prime}$ is a union of cones, $C$ is disjoint from $A^{\prime}$ and that $1 \leqslant\left|B \backslash A^{\prime}\right| \leqslant \operatorname{Ex}(B)$. We will show that $w_{-1}=(-1,-1), w_{0}=(0,-1)$ and $w_{1}=(1,-1)$ are in $A$. By symmetry, we need only show $w_{0}$ and $w_{1}$ are in $A$.

$w_{0} \in A$. If $w_{0} \notin A$, then $w_{0}$ is contained in a cone $C_{0}$ disjoint from $A$, which we may assume is at $w_{0}$. Since $v \notin C_{0}, C_{0}$ must either be below, to the left or to the right of $w_{0}$. In either of the latter two cases, $C_{0} \cup C$ contains either the intersection of the extremal line $y-x=b$ with $B$ or the intersection of the extremal line $y+x=d$ with $B$. As, $\left|\left(C_{0} \cup C\right) \cap B\right| \leqslant\left|B \backslash A^{\prime}\right| \leqslant \operatorname{Ex}(B)$, this contradicts Lemma 5.10(2). Thus we may assume that $C_{0}$ is the cone below $w_{0}$.

Note that $v$ does not lie on the extremal line $y-x=a$ nor the extremal line $y+x=c$, for otherwise $C$ contains the intersection of an extremal line with $B$ and we get a contradiction as in the previous paragraph. Let $L$ be the line of slope -1 passing through $v$. Let $\phi: X^{0} \rightarrow X^{0}$ be the translation $(x, y) \mapsto(x-1, y)$. As $v$ does not lie on $y-x=a$ or $y+x=c$, given a vertex $u \in L \cap B$ it follows that either $u \in C \cap B \cap L$ or $\phi(u) \in C_{0} \cap B$. It follows that

$$
|B \cap L| \leqslant|C \cap B \cap L|+\left|C_{0} \cap B \cap L\right| \leqslant|C \cap B|+\left|C_{0} \cap B\right| \leqslant\left|B \backslash A^{\prime}\right| \leqslant \operatorname{Ex}(B) .
$$

As $v$ does not lie on $y-x=a$ or $y+x=c$, either $L$ is an extremal line of $B$ or $(0,1) \in B$. If $L$ is an extremal line, then this contradicts Lemma 5.10(2). If not, then $(0,1) \in C \cap(B \backslash L)$, so $|C \cap L \cap B|<|C \cap B|$. Thus the inequality in (7.1) is strict, contradicting Lemma 5.10(3). We deduce that $w_{0} \in A$.

$w_{1} \in A$. Suppose $w_{1} \notin A$. Then there is a cone $C_{1}$ based at $w_{1}$ that does not intersect $A$. Since $v \notin C_{1}, C_{1}$ must be either below or to the right of $w_{1}$. In either case, if $L$ is the line through $v$ and $w_{1}$, then $B \cap L \subseteq C \sqcup C_{1}$ and so

$$
|B \cap L| \leqslant\left|C_{1} \cap B \cap L\right|+|C \cap B \cap L| \leqslant\left|C_{1} \cap B\right|+|C \cap B| \leqslant\left|B \backslash A^{\prime}\right| \leqslant \operatorname{Ex}(B) .
$$


If $L$ an extremal line of $B$, then (7.2) contradicts Lemma 5.10(2). We thus assume $L$ is not an extremal line of $B$.

We claim that the inequality $|B \cap L| \leqslant \operatorname{Ex}(B)$ above is strict. If we show this, then we get a contradiction by Lemma $5.10(3)$, and we can deduce that $w_{1} \in A$ as required. We first observe that as $L$ is not an extremal line of $B$ and as $w_{0}=(0,-1) \in B$ (by what we have already shown), we must have that $(1,0) \in B$. There are now two cases depending on whether or not $w_{1} \in B$. If $w_{1} \notin B$, then the line $y-x=-1=a \operatorname{through}(0,-1)$ and $(1,0)$ is an extremal line of $B$. Furthermore, as $\operatorname{Ex}(B)>0$, it follows from Lemma $5.10(2)$ that $b-a \geqslant 2$. Thus, we conclude that $(0,1) \in(B \cap C) \backslash L$. We thus deduce that $|C \cap L \cap B|<|C \cap B|$ and that the inequality in (7.2) is strict, proving the claim when $w_{1} \notin B$.

If $w_{1} \in B \backslash A$, then $\operatorname{Ex}(B) \geqslant|B \backslash A|+1 \geqslant 2$. Lemma 5.10(2) then implies that every extremal line of $B$ contains at least three vertices, and so $b-a, d-c \geqslant 4$. As $v, w_{1},(0,-1),(1,0) \in B$, it follows that $B \backslash L$ must intersect at least one of $C$ or $C_{1}$, and so either $|C \cap L \cap B|<|C \cap B|$ or $\left|C_{1} \cap L \cap B\right|<\left|C_{1} \cap B\right|$. In either case, we deduce as before that the inequality in (7.2) is strict as required.

Lemma 7.5. Up to congruence, $B(0,2)$ is the only disconnected box that is a minimal set. In particular, if $B$ is a box with $\operatorname{Ex}(B)>0$, then $B$ is connected.

Proof. Suppose $B$ is a disconnected box that is a minimal set. Then it contains more than one vertex and is contained in a standard line. Thus $B$ is congruent to $B(0,2 n)$ for some $n>0$. Note that $\operatorname{Ex}(B)=\frac{n(1-n)}{2}$ by Theorem 5.13. Lemma 4.3 implies $n=1$ and so $B=B(0,2)$. Furthermore, as $\operatorname{Ex}(B)=0$, the second claim follows.

The following lemma is the inductive step in our proof of Proposition 7.7.

Lemma 7.6. Let $A$ be a connected minimal set such that $X^{0} \backslash A$ is a union of cones. Suppose $A^{\prime} \subseteq A$ is also minimal, $\operatorname{enc}\left(A^{\prime}\right)=\operatorname{enc}(A)$ and $A \backslash A^{\prime}=\{v\}$. Then $A^{\prime}$ is connected and there exists a cone $C$ based at $v$ such that $C \cap A^{\prime}=\emptyset$. In particular, $X^{0} \backslash A^{\prime}$ is also a union of cones.

Proof. Since $A$ and $A^{\prime}$ are minimal and enc $(A)=\operatorname{enc}\left(A^{\prime}\right)$, Lemma 6.3 ensures $|\partial A|=\left|\partial A^{\prime}\right|$. As $|\partial A|=\left|\partial A^{\prime}\right|, A^{\prime} \subset A,|A|=\left|A^{\prime}\right|+1$ and $A$ is connected, there exists some vertex $w \in \partial A$ adjacent to $v$ which is not in $N\left(A^{\prime}\right)$. In particular, $w$ is adjacent to $v$ and no other vertex in $A$. Without loss of generality, we can suppose $v=(0,0)$ and $w=(0,1)$. Suppose $R^{+}$and $R^{-}$are the rays $\{(x, y) \mid y-x=0, y \geqslant 1\}$ and $\{(x, y) \mid y+x=0, y \geqslant 1\}$ respectively. Since $b:=(1,1)$ is adjacent to $w$, it is not contained in $A$. As $X^{0} \backslash A$ is a union of cones, $b$ is contained in a cone $C^{\prime}$ that is disjoint from $A$. Since $C^{\prime}$ cannot contain $v$, it must contain the ray $R^{+}$, so that $R^{+} \cap A=\emptyset$. A similar argument using $(-1,1)$ allows one to deduce $R^{-} \cap A=\emptyset$. Let $C$ be the cone above $v$. As $A$ is connected and $\{w\} \cup R^{-} \cup R^{+}$do not contain vertices of $A$, it follows that $C \cap A^{\prime}=\emptyset$. Since $X \backslash A$ is a union of cones, $X \backslash A^{\prime}=C \cup(X \backslash A)$ is also a union of cones.

Let $B:=\operatorname{enc}(A)=\operatorname{enc}\left(A^{\prime}\right)$. Since $A^{\prime}$ is minimal, we have $\left|B \backslash A^{\prime}\right| \leqslant \operatorname{Ex}(B)$, and so $|B \backslash A| \leqslant \operatorname{Ex}(B)-1$. Thus Lemma 7.4 can be applied to $A, v$ and $C$ to deduce 
that $(-1,-1),(0,-1),(1,-1) \in A^{\prime}$. We now show $A^{\prime}$ is connected. We pick arbitrary $p, q \in A^{\prime}$ and show that $p$ and $q$ can be joined by a path in $A^{\prime}$. Since $A$ is connected, there exists a simple path $P=\left(p=v_{0}, v_{1}, \ldots, q=v_{m}\right)$ in $A$. If $P$ is a path in $A^{\prime}$ we are done. If not, then $v_{i}=(0,0)$ for some $0<i<m$. Since $P$ is simple, $v_{j} \in A^{\prime}$ for all $j \neq i$. Thus $v_{i-1}$ and $v_{i+1}$ are contained in $A^{\prime}$ and are adjacent to $v$, and so $v_{i-1}, v_{i+1} \in\{(-1,0),(1,0),(0,-1)\}$. Since $(-1,-1),(0,-1),(1,-1) \in A^{\prime}, v_{i-1}$ and $v_{i+1}$ can be joined by a path $\left(v_{i-1}=u_{0}, u_{1}, \ldots, u_{t}=v_{i+1}\right)$ in $A^{\prime}$. Thus $\left(p=v_{0}, \ldots, v_{i-1}=\right.$ $\left.u_{0}, \ldots, u_{t}=v_{i+1}, \ldots v_{m}=q\right)$ is a path from $p$ to $q$ in $A^{\prime}$.

The next proposition describes the geometry of a minimal set.

Proposition 7.7. Suppose $A \subset X^{0}$ is minimal and $n:=|\operatorname{enc}(A) \backslash A|>0$. Then there exists a nested sequence $A=A_{0} \subset A_{1} \subset \cdots \subset A_{n}=\operatorname{enc}(A)$ of connected minimal sets such that $\left|A_{i}\right|=\left|A_{i-1}\right|+1$ with the following property. For $i>0$ and $v_{i} \in A_{i} \backslash A_{i-1}$, there exists a cone $C_{i}$ based at $v_{i}$ such that $C_{i} \cap A_{i-1}=\left\{v_{i}\right\}$. In particular, $A$ is connected and $X^{0} \backslash A$ is a union of cones.

Proof. Since $n>0, \operatorname{Ex}(\operatorname{enc}(A))>0$ and so enc $(A)$ is connected by Lemma 7.5. By Lemma 6.3, there exists a nested sequence $A=A_{0} \subset A_{1} \subset \cdots \subset A_{n}=\operatorname{enc}(A)$ of minimal sets such that $\left|A_{i+1}\right|=\left|A_{i}\right|+1$. Since $A_{n}=\operatorname{enc}(A)$ is connected and $X^{0} \backslash A_{n}$ is a union of cones (by Remark 7.3), we can apply Lemma 7.6 successively to give the desired conclusion.

Proof of Theorem 7.1. Suppose $A$ is a minimal set that is not connected. Proposition 7.7 implies that $A=\operatorname{enc}(A)$. By Lemma $7.5, A$ is congruent to $B(0,2)$. Since $\operatorname{Ex}(B(0,2))=0$, Theorem 6.4 ensures $B(0,2)$ is indeed minimal.

\subsection{A characterization of minimal sets using cones}

We can refine Theorem 6.4 by providing an additional characterization of minimal sets using cones.

Theorem 7.8. Let $A \subset X^{0}$ with $N:=|\operatorname{enc}(A) \backslash A|$ and $E:=\operatorname{Ex}(\operatorname{enc}(A))$. Then the following are equivalent:

1. A is minimal;

2. $|\partial A|=|\partial(\operatorname{enc}(A))|$ and $N \leqslant E$;

3. $X^{0} \backslash A$ is a union of cones and $N \leqslant E$.

Proof. The equivalence of (1) and (2) follows from Theorem 6.4. Theorem 6.4 and Proposition 7.7 tell us that (1) implies (3). All that remains is to show (3) implies (1).

Let $A \subset X^{0}$ with $|B \backslash A| \leqslant \operatorname{Ex}(B)$, where $B:=\operatorname{enc}(A)$. Suppose $X^{0} \backslash A$ is a union of cones. Since $\operatorname{Ex}(B) \geqslant 0, B$ is minimal by Lemma 4.3. Let $D$ be a minimal set such that $A \subset D \subseteq B$, with $|D|$ minimal among all such choices. Notice that enc $(D)=B$.

For contradiction, suppose $A$ is not minimal. Then $A \subsetneq D$, so pick $v \in D \backslash A$. As $X^{0} \backslash A$ is a union of cones, there exists a cone $C^{\prime}$ containing $v$ and disjoint from $A$. Without 
loss of generality, we may suppose $C^{\prime}$ faces upwards (i.e. it lies above some vertex) and that $v \in D \cap C^{\prime}$ has maximal $y$-coordinate out of all vertices in $D \cap C^{\prime}$. Let $C$ be the cone above $v$. Since $C \subset C^{\prime}$ and $v$ has maximal $y$ coordinate, $C$ is a cone above $v$ such that $C \cap D=\{v\}$.

Since $D$ is a minimal set, Proposition 7.7 ensures $X^{0} \backslash D$ is a union of cones. Moreover, since $A \subsetneq D \subseteq B,|B \backslash D|<|B \backslash A| \leqslant \operatorname{Ex}(B)$. Without loss of generality, we can assume that $v=(0,0)$. Lemma 7.4 now implies that $(-1,-1),(0,-1)$ and $(1,-1)$ are in $D \backslash\{v\}$. As $(-1,-1),(0,-1),(1,-1) \in D$ and $C \cap D=\{v\}$, it follows that $|\partial(D \backslash\{v\})|=|\partial D|$. Theorem 6.4 thus ensures $D \backslash\{v\}$ is minimal. Since $A \subseteq D \backslash\{v\} \subsetneq D \subseteq B$, this contradicts our choice of $D$. Thus $A$ is minimal as required.

\subsection{Neighborhoods of minimal sets are minimal}

We recall that if $A \subseteq X^{0}$, then its neighborhood $N(A)$ is defined to be the set $A \cup \partial A$. The aim of this section is to prove the following:

Theorem 7.9. If $A \subseteq X^{0}$ is minimal, then $N(A)$ is also minimal.

We will use Theorem 7.8 to prove Theorem 7.9. We first analyze the excess of enc $(N(A))$ and the difference $N^{\prime}=|\operatorname{enc}(N(A)) \backslash N(A)|$.

Lemma 7.10. For a finite set $A \subset X^{0}$, enc $(N(A))=N(\operatorname{enc}(A))$.

Proof. Suppose enc $(A)=B(a, b, c, d)$. We first observe that $N(\operatorname{enc}(A))$ is the box $B^{\prime}:=$ $B(a-1, b+1, c-1, d+1)$. As $A \subseteq \operatorname{enc}(A)$, we have $N(A) \subseteq N(\operatorname{enc}(A))=B^{\prime}$ and so enc $(N(A)) \subseteq B^{\prime}$. Since $A$ intersects all four extremal lines of enc $(A)$, it follows $N(A)$ intersects all four extremal lines of $B^{\prime}$, and so $B^{\prime} \subseteq \operatorname{enc}(N(A))$. Therefore $B^{\prime}=$ $\operatorname{enc}(N(A))=N(\operatorname{enc}(A))$.

Lemma 7.11. Let $A$ be a minimal set. Then

$$
\begin{aligned}
& \text { 1. }|\operatorname{enc}(N(A)) \backslash N(A)|=|\operatorname{enc}(A) \backslash A| . \\
& \text { 2. } \operatorname{Ex}(\operatorname{enc}(N(A)))=\operatorname{Ex}(\operatorname{enc}(A))+1
\end{aligned}
$$

Proof. Since $A$ is minimal, by Theorem 6.4 we have $|\partial A|=|\partial \operatorname{enc}(A)|$. By Lemma 7.10 we have

$$
\begin{aligned}
|\operatorname{enc}(N(A)) \backslash N(A)| & =|\operatorname{enc}(N(A))|-|N(A)| \\
& =|N(\operatorname{enc}(A))|-|N(A)| \\
& =(|\operatorname{enc}(A)|+|\partial \operatorname{enc}(A)|)-(|A|+|\partial A|) \\
& =|\operatorname{enc}(A)|-|A| \\
& =|\operatorname{enc}(A) \backslash A| .
\end{aligned}
$$

For the proof of part (2), suppose enc $(A)$ is congruent the box $B(\alpha, \beta)$ (or $\hat{B}(\alpha, \beta))$. Using Lemma 7.10, we get enc $(N(A))$ is congruent to $B(\alpha+2, \beta+2)($ or $\hat{B}(\alpha+2, \beta+2))$. Then by the formula for the excess of a box in Theorem 5.13, we get $\operatorname{Ex}(\operatorname{enc}(N(A)))=$ $\operatorname{Ex}(\operatorname{enc}(A))+1$. 
For a cone $C$ in $X$, let $\stackrel{\circ}{C}$ denote the collection of points not contained in any extremal line of $C$. Note that $\dot{C}$ is itself a cone.

Lemma 7.12. Let $A \subset X^{0}$ be a minimal set. Suppose $v \in A$ and $C$ is a cone based at $v$

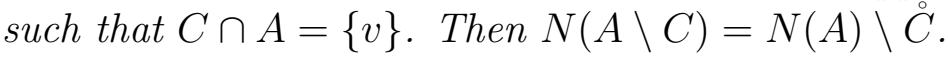

Proof. Without loss of generality, we can suppose $v=(0,0)$ and that $C$ is the cone above $v$. By Lemma $7.4,(-1,-1),(0,-1),(1,-1) \in A$. Suppose $x \in N(A \backslash C)$. Then $x$ is adjacent

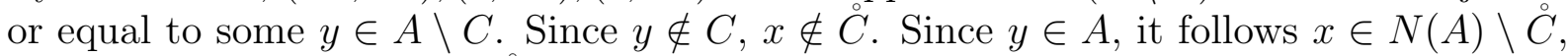
and so $N(A \backslash C) \subseteq N(A) \backslash \stackrel{\circ}{C}$.

Conversely, suppose $x \in N(A) \backslash \stackrel{\circ}{ }$. Then $x$ is adjacent or equal to some $y \in A$. If $y \notin C$, then $x \in N(A \backslash C)$. If $y \in C$, then $y=(0,0)$. Since $(0,1) \in \dot{C}, x$ is equal to one of $(-1,0)$, $(0,-1)$ or $(1,0)$. In all three cases, $x$ is adjacent to one of $(-1,-1),(0,-1),(1,-1) \in A \backslash C$

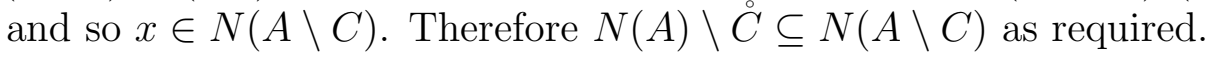

Proposition 7.13. If $A \subset X^{0}$ is a minimal set, then $X^{0} \backslash N(A)$ is a union of cones.

Proof. By Proposition 7.7, there is a nested sequence $A=A_{0} \subset A_{1} \subset \cdots \subset A_{n}=\operatorname{enc}(A)$ of minimal sets and a sequence $C_{1}, \ldots, C_{n}$ of cones such that $A_{i} \backslash A_{i-1}=\left\{v_{i}\right\}$ and $C_{i}$ is based at $v_{i}$ with $C_{i} \cap A_{i}=\left\{v_{i}\right\}$. In particular, $A=\operatorname{enc}(A) \backslash \cup_{i=1}^{n} C_{i}$. By successively applying Lemma 7.12 and using Lemma 7.10, we see that

$$
\begin{aligned}
N(A) & =N\left(\operatorname{enc}(A) \backslash \cup_{i=1}^{n} C_{i}\right) \\
& =N(\operatorname{enc}(A)) \backslash \cup_{i=1}^{n} \stackrel{\circ}{C}_{i} \\
& =\operatorname{enc}(N(A)) \backslash \cup_{i=1}^{n} \stackrel{\circ}{C}_{i}
\end{aligned}
$$

As $N(A)=\operatorname{enc}(N(A)) \backslash \cup_{i=1}^{n} \stackrel{\circ}{C}_{i}$ and by Remark 7.3, $X^{0} \backslash N(A)$ is a union of cones.

Proof of Theorem 7.9. Combine Lemma 7.11, Proposition 7.13 and Theorem 7.8.

\section{The graph of minimal sets}

In this section, we study the graph of minimal sets, $\mathcal{G}$ (see the introduction for a definition).

\subsection{Dead and mortal sets}

A finite subset $A \subset X^{0}$ is efficient if for every $B \subset X^{0},|\partial B|=|\partial A|$ implies $|A| \geqslant|B|$, and we say that $A$ is inefficient otherwise. Equivalently, $A$ is efficient if $|\partial B|=|\partial A|$ implies that $\operatorname{Ex}(A) \geqslant \operatorname{Ex}(B)$ by Lemma 4.4. The main result of this subsection is Theorem 8.5, which characterizes dead sets (defined in the introduction) as inefficient sets, which in turn are classified in terms of specific boxes (Lemma 8.3), and Theorem 8.6 characterizing mortal sets.

We first show that efficient sets are boxes and are minimal:

Lemma 8.1. Every efficient set is minimal, saturated and a box. 
Proof. Let $A$ be an efficient set. By Lemma 4.2, there exists a minimal set $A^{\prime}$ such that $\left|\partial A^{\prime}\right|=|\partial A|$. Since $A$ is efficient, we have $|A| \geqslant\left|A^{\prime}\right|$. If $A$ is not minimal, then there exists a minimal set $A^{\prime \prime}$ such that $\left|A^{\prime \prime}\right|=|A| \geqslant\left|A^{\prime}\right|$ and $\left|\partial A^{\prime \prime}\right|<|\partial A|=\left|\partial A^{\prime}\right|$. This contradicts Lemma 2.1, so $A$ must be minimal. If $A$ is not saturated, then there exists a vertex $v$ such that $|\partial(A \cup\{v\})| \leqslant|\partial A|$, which contradicts Lemma 2.1 and the fact that $A$ is minimal and efficient. Proposition 6.1 now implies that every efficient set is a box.

We say $A \subset X^{0}$ is a Wang-Wang box if it is simultaneously a box and a Wang-Wang set.

Remark 8.2. A subset of $X^{0}$ is a Wang-Wang box if and only if it is congruent to either $B(m, m)$ or $B(m, m+1)$ for some $m \in \mathbb{Z}$ (recall Notation 5.5).

We now characterize efficient sets.

Lemma 8.3. A subset of $X^{0}$ is efficient if and only if it is congruent to either a WangWang box or $B(m-1, m+1)$ for some odd $m \in \mathbb{N}$.

Proof. Let $B$ be an efficient set. By Lemma 8.1, $B$ is a box. Remark 5.9 implies that no box of the form $\hat{B}(\alpha, \beta)$ is efficient. It thus follows from Proposition 5.6 that $B$ is congruent to $B(\alpha, \beta)$ for some $\alpha, \beta \in \mathbb{Z}$. Without loss of generality, we may assume that $\beta \geqslant \alpha$, and we set $r=\frac{\alpha+\beta}{2}$ and $k=\frac{\beta-\alpha}{2}$. Note that $\alpha=r-k$ and $\beta=r+k$.

Suppose first that $\alpha$ and $\beta$ have the same parity. Then $r$ and $k$ are both integers. By Lemma 5.7, $|\partial B(r-k, r+k)|=|\partial B(r, r)|$. Since $B$ is efficient, Theorem 5.13 gives:

$$
\operatorname{Ex}(B(r-k, r+k))=\left\lfloor\frac{\lfloor r\rfloor-k^{2}}{2}\right\rfloor \geqslant \operatorname{Ex}(B(r, r))=\left\lfloor\frac{\lfloor r\rfloor}{2}\right\rfloor .
$$

By the above equation, either $k=0$, or $r$ is odd and $k=1$. Thus $B$ is either the Wang-Wang box $B(\alpha, \alpha)$ or the box $B(m-1, m+1)$ for $m$ odd, respectively.

On the other hand, suppose $\alpha$ and $\beta$ have different parity. Then $r=s+\frac{1}{2}$ and $k=t+\frac{1}{2}$ for some $s, t \in \mathbb{N}$. By Lemma 5.7, $|\partial B(r-k, r+k)|=|\partial B(r-1 / 2, r+1 / 2)|$. By applying Theorem 5.13 and using that $B$ is efficient and $t^{2}+t$ is even, we get the following:

$\operatorname{Ex}(B(r-k, r+k))=\left\lfloor\frac{s-t^{2}-t-\frac{1}{4}}{2}\right\rfloor=\left\lfloor\frac{s-\frac{1}{4}}{2}\right\rfloor-\frac{t^{2}+t}{2} \geqslant \operatorname{Ex}(B(r-1 / 2, r+1 / 2)) \geqslant\left\lfloor\frac{s-\frac{1}{4}}{2}\right\rfloor$

Thus $t^{2}+t \leqslant 0$, which implies $t=0$ and hence $\beta=\alpha+1$. Thus $B$ is congruent to $B(\alpha, \alpha+1)$, which is a Wang-Wang box.

For the converse, suppose we are given a Wang-Wang box $W W_{n}$. By Lemma 5.3, a box is saturated. This implies $\left|\partial W W_{n+1}\right|>\left|\partial W W_{n}\right|$. Since $W W_{n+1}$ is minimal, Lemma 2.1 ensures that given any $B \subset X^{0}$ with $|B|>\left|W W_{n}\right|$ then $|\partial B|>\left|\partial W W_{n}\right|$. Thus a WangWang box $W W_{n}$ is efficient. By Lemma 5.7 and Theorem 5.13, for odd $m$, we have $|\partial B(m-1, m+1)|=|\partial B(m, m)|$ and $\operatorname{Ex}(B(m-1, m+1))=\operatorname{Ex}(B(m, m))$. As $B(m, m)$ is efficient, $B(m-1, m+1)$ is also.

Before proving the next result, we first show that one can always add a vertex to a box such that the resulting set has boundary one larger than the box, as long as the box contains at least two vertices. 
Lemma 8.4. Let $B \subset X^{0}$ be a box containing at least two vertices. Then $|\partial(B \cup\{v\})|=$ $|\partial B|+1$ for some $v \in X^{0} \backslash B$.

Proof. Let $(x, y)$ be a vertex of $B$ with $y$ maximal. Suppose first that $(x+1, y) \in B$. Since $B$ is saturated by Lemma 5.3 , it follows from Lemma 3.2 that $(x-1, y) \notin B$. The claim now follows for this case by noting that $|\partial(B \cup\{(x, y+1)\})|=|\partial B|+1$. A similar argument shows the claim when $(x-1, y) \in B$.

Next consider the case where $(x-1, y) \notin B$ and $(x+1, y) \notin B$. Suppose first that $(x, y-1) \in B$. As $B$ is a box with $(x, y) \in B$ and $(x+1, y) \notin B$, it follows that $(x+2, y) \notin B \cup \partial B$. The claim now follows by noting that $|\partial(B \cup\{(x+1, y)\})|=|\partial B|+1$. Finally, suppose that $(x, y-1) \notin B$. In this case, as $B$ is a box and $|B| \geqslant 2$, we must have that either $(x-1, y-1) \in B$ or $(x+1, y-1) \in B$. Without loss of generality, suppose the former is true. As $B$ is saturated, Lemma 3.2 implies that $(x-2, y-1) \notin B$. It now follows that $|\partial(B \cup\{(x-1, y)\})|=|\partial B|+1$.

We now give a characterization of dead sets.

Theorem 8.5. Let $A \subset X^{0}$ be a minimal set. The following are equivalent:

1. A is dead.

2. A is an inefficient box.

3. $A$ is a box that is not congruent to either a Wang-Wang box or to a box of the form $B(m-1, m+1)$ for odd $m \in \mathbb{Z}$.

Proof. Suppose $A$ is dead. We first show that $A$ is saturated. Let $v \in X^{0} \backslash A$. If $|\partial(A \sqcup\{v\})| \leqslant|\partial A|$, then Lemma 2.1 would imply that $A \sqcup\{v\}$ is minimal, contradicting the hypothesis that $A$ is dead. Thus $|\partial(A \sqcup\{v\})|>|\partial A|$ for all $v \in X^{0} \backslash A$, ensuring that $A$ is saturated. Since $A$ is saturated and minimal, Proposition 6.1 implies it is a box. Since a set with one vertex is not dead, $|A|>1$. Hence Lemma 8.4 ensures that there exists a vertex $v \in X^{0} \backslash A$ such that $|\partial(A \cup\{v\})|=|\partial A|+1$. Since $A$ is dead, $A \cup\{v\}$ is not minimal and so there exists a minimal set $C$ such that $|C|=|A|+1$ and $|\partial C|<|\partial A|+1$. As $A$ is minimal, Lemma 2.1 implies $|\partial C|=|\partial A|$. Thus $A$ is an inefficient box.

Now suppose $A$ is an inefficient box. Since $A$ is saturated by Lemma 5.3, $|\partial(A \cup\{v\})|>$ $|\partial A|$ for every $v \in X^{0} \backslash A$. As $A$ is inefficient, there exists a set $C$ such that $|C|>|A|$ and $|\partial C|=|\partial A|$. Lemma 2.1 now implies that $A \cup\{v\}$ isn't minimal for any $v \in X^{0} \backslash A$. Hence, $A$ is dead. The equivalence of (2) and (3) is shown in Lemma 8.3.

Finally, we characterize mortal sets:

Theorem 8.6. A minimal set is mortal if and only if its enclosing box is dead.

Proof. Let $A$ be a minimal set. We first show that $A$ is mortal if and only if $\operatorname{enc}(A)$ is mortal. By Lemma 6.3, there exists a sequence $A=A_{0} \subset A_{1} \subset \cdots \subset$ enc $(A)$ of nested minimal sets such that $\left|A_{i+1}\right|=\left|A_{i}\right|+1$. Thus, if $A$ is mortal, so is enc $(A)$. On the other hand, suppose $\operatorname{enc}(A)$ is mortal. By Lemma 6.3, any maximal nested sequence 
$A=A_{0}^{\prime} \subset A_{1}^{\prime} \subset \ldots$ of minimal sets with $\left|A_{i+1}^{\prime}\right|=\left|A_{i}^{\prime}\right|+1$ must include enc $(A)$ and, in particular, must be finite as $\operatorname{enc}(A)$ is mortal. Thus, $A$ is mortal.

Consequently, in order to prove the theorem, we need to show that a box $B$ is mortal if and only if it is dead. Since dead sets are mortal, this reduces to demonstrating that a box which is not dead is immortal. By Theorem 8.5 we only need to show that Wang-Wang boxes and $B(m-1, m+1)$, for odd $m \in \mathbb{Z}$, are immortal sets. Wang-Wang boxes are immortal because they are contained in the infinite nested sequence of minimal sets $\left(W W_{n}\right)_{n=1}^{\infty}$.

Let $B=B(m-1, m+1)$ for some odd $m \in \mathbb{Z}$. Let $v=(0, m)$ and $B^{\prime}:=B \sqcup\{v\}$. Since $v$ is not contained in $B$ but is adjacent to $(0, m-1),(1, m) \in B$, it follows that $\left|\partial B^{\prime}\right|=|\partial B|+1$. By Lemma 5.7, Lemma 5.8 and as $m$ is odd, $|B|=|B(m, m)|$ and $|\partial B|=|\partial B(m, m)|$. Since $B(m, m)$ is a Wang-Wang box and in particular, it is saturated, then any minimal set of size $|B|+1$ must have boundary strictly greater than $|\partial B|$. Thus, $B^{\prime}$ is a minimal set. By Lemma 6.3, there exists a sequence of minimal sets $B \subset B^{\prime} \subset \cdots \subset \operatorname{enc}\left(B^{\prime}\right)$ such that the size of the symmetric difference between consecutive sets in this sequence is one. As enc $\left(B^{\prime}\right)=B(m, m+1)$ is a Wang-Wang set (see Remark 8.2), it is immortal. Thus, $B$ is immortal as well.

\subsection{Uniquely minimal sets}

In this subsection, we characterize uniquely minimal sets in $X$. Recall from the introduction that the grading of a vertex of $\mathcal{G}$ is the size of one of its representatives, and uniquely minimal sets correspond exactly to vertices of $\mathcal{G}$ that are unique out of vertices of the same grading.

Lemma 8.7. Let $W W_{n}$ be a Wang-Wang set that is not a box. Then there exists a minimal set $A$ such that $|A|=n$ and $A$ is not congruent to $W W_{n}$.

Proof. The box $B:=\operatorname{enc}\left(W W_{n}\right)$ is congruent to $B(\alpha, \beta)$ where either $\alpha=\beta$ or $\alpha+1=\beta$. By Lemma 5.12 and Theorem 6.4, $k:=|B|-\left|W W_{n}\right| \leqslant \operatorname{Ex}(B) \leqslant \frac{\alpha}{2}$. Since $k \geqslant 1$ (as $W W_{n}$ is not a box), Lemma 5.12 implies that either $\alpha \geqslant 3$ or $\alpha=\beta=2$ (indeed, $\operatorname{Ex}(B(2,3))=0)$. In the latter case, $k=1$ and $n=4$, so we observe that $|\hat{B}(2,2)|=\left|W W_{4}\right|$ and $|\partial \hat{B}(2,2)|=\left|\partial W W_{4}\right|$. Since $\hat{B}(2,2)$ is not congruent to $W W_{4}$, we are done.

Therefore, we may assume $\alpha \geqslant 3$. Let $B^{\prime}:=B(\alpha-1, \beta+1)$. By Lemma 5.7, $|\partial B|=\left|\partial B^{\prime}\right|$. By Lemma 5.8,

$$
\begin{gathered}
|B|=\left\lfloor\frac{\alpha \beta+\alpha+\beta+2}{2}\right\rfloor \\
\left|B^{\prime}\right|=\left\lfloor\frac{(\alpha-1)(\beta+1)+\alpha+\beta+2}{2}\right\rfloor=\left\lfloor\frac{\alpha \beta+2 \alpha+1}{2}\right\rfloor .
\end{gathered}
$$

Thus, we have that $|B|-1 \leqslant\left|B^{\prime}\right| \leqslant|B|$ in both the case that $\alpha=\beta$ and that $\beta=\alpha+1$.

Note that the line $y=x$ contains $\left\lfloor\frac{\beta+1}{2}+1\right\rfloor$ vertices of $B^{\prime}$. Since $k \leqslant \frac{\alpha}{2}<\frac{\beta+1}{2}$, by Lemma 5.10 there exists some set $A$ such that $|\partial A|=|\partial B|=\left|\partial W W_{n}\right|,|A|=|B|-k=$ $\left|W W_{n}\right|$, and enc $(A)=B^{\prime}$. In particular, $A$ must be minimal. Since $B^{\prime}=\operatorname{enc}(A)$ and $B=\operatorname{enc}\left(W W_{n}\right)$ are not congruent, $A$ and $W W_{n}$ are not congruent. 
Theorem 8.8. A subset of $X^{0}$ is uniquely minimal if and only if it is congruent to either $B(2 n, 2 n)$ or $B(n, n+1)$ for some $n \in \mathbb{N}$.

Proof. Suppose $A$ is uniquely minimal. Since $W W_{|A|}$ is minimal and $\left|W W_{|A|}\right|=|A|, A$ must be congruent to $W W_{|A|}$. By Lemma 8.7, $A$ must be a box. We note that $A$ cannot be congruent to $B(r, r)$ for odd $r \in \mathbb{N}$, since Lemma 5.7 and Lemma 5.8 imply that $|B(r-1, r+1)|=|B(r, r)|$ and $|\partial B(r-1, r+1)|=|\partial B(r, r)|$. Thus $A$ is a box of the form $B(2 n, 2 n)$ or $B(n, n+1)$ for some $n \in \mathbb{N}$ by Remark 8.2.

For the converse, suppose $B$ is congruent to $B(2 n, 2 n)$ or $B(n, n+1)$ for some $n \in \mathbb{N}$. In particular, $B$ is congruent to a Wang-Wang set, so it is minimal. Suppose $A$ is another minimal set such that $|A|=|B|$. It follows that $|\partial A|=|\partial B|$. By Lemma 8.3 $B$ is efficient. Since $|A|=|B|$, we get that $A$ is also efficient. Furthermore, Lemma 8.3 also implies that any efficient set of size $|B|$ is actually congruent to $B$, hence $A$ is congruent to $B$.

Corollary 8.9. The graph $\mathcal{G}$ contains exactly one infinite connected component.

Proof. Let $\mathcal{C}$ be an infinite component of $\mathcal{G}$. As there are only finitely many sets (up to congruence) of any given size, there exists a number $m_{0}$ such that $\mathcal{C}$ contains a vertex of grading $m$ for every $m \geqslant m_{0}$. By Theorem $8.8, B(2 n, 2 n)$ is uniquely minimal for every $n$. Thus, $\mathcal{C}$ contains $B(2 n, 2 n)$ for every $n$ sufficiently large, and so $\mathcal{C}$ is the unique infinite component of $\mathcal{G}$.

\subsection{Finite components}

In this subsection, we show that $\mathcal{G}$ contains infinitely many isolated vertices and finite components with arbitrarily many vertices. To do so, we prove the following more general result that gives sufficient conditions for a vertex of $\mathcal{G}$ to be contained in a finite component, i.e. a component of $\mathcal{G}$ that is a finite subgraph. Moreover, the possible gradings of vertices in this component is exactly described. Recall from the introduction that the height of a component is the maximal length of a nested sequence of minimal sets in it.

Proposition 8.10. Let $B \subset X$ be a box such that:

1. $\operatorname{Ex}(B)=d \geqslant 0$

2. The modulus $\{\alpha, \beta\}$ of $B$ satisfies $\alpha, \beta \geqslant 2$

3. Given any standard line $L, B \cap L$ is either empty or contains at least $d+2$ vertices.

4. $B$ is a dead set

Let $\mathcal{C}$ be the component of $\mathcal{G}$ containing the vertex representing $B$. Then, for any vertex in $\mathcal{C}$ representing a set $A$, we have that $|B|-d \leqslant|A| \leqslant|B|$ and that $\operatorname{enc}(A)$ is congruent to $B$. Furthermore, $\mathcal{C}$ contains a vertex representing a set of size $k$ for every $|B|-d \leqslant k \leqslant|B|$. In particular, $\mathcal{C}$ is finite and has height exactly $d+1$. 
Proof. We first prove the final claim. Let $B=B_{0} \supset B_{1} \supset \cdots \supset B_{n}$ be the sets given by Lemma 5.10 where $\left|\partial B_{i}\right|=|\partial B|$ and $n=|L \cap B|-1 \geqslant d+1$ (where $L$ is an extremal line of $B$ ). As $\operatorname{Ex}(B)=d$, we have that $B_{i}$ is minimal for each $i \leqslant d$. The claim follows.

Now let $\mathcal{C}^{\prime}$ be the set of all vertices in $\mathcal{C}$ represented by a set $C$ such that there exists a path $C=C_{0}, \ldots, C_{n}=B$ in $\mathcal{G}$ with $n \leqslant d$ and $\left|C_{i+1}\right|=\left|C_{i}\right|+1$ for all $0 \leqslant i<n$. As $\operatorname{Ex}(B)=d$, for all $C \in \mathcal{C}^{\prime}$ we must have that $|\partial C|=|\partial B|$ and, consequently by Lemma 6.3, we have that enc $(C)=B$. Thus, to prove the remaining claims of the theorem, it is enough to show that $\mathcal{C}^{\prime}=\mathcal{C}$. Additionally, as $\mathcal{C}$ is connected, it is enough to show that given any vertex $v$ in $\mathcal{G}$, represented by a set $A$, that is adjacent to a vertex in $\mathcal{C}^{\prime}$, represented by a set $C$, then $v \in \mathcal{C}^{\prime}$. Let $A$ and $C$ be such sets.

Suppose first that $A \subset C$. Then $|A|=|C|-1 \geqslant|B|-d-1$ (by the definition of $\mathcal{C}^{\prime}$ ) and by $(3)$ it follows that $A$ contains a vertex in every standard line which has non-empty intersection with $B$. Thus $\operatorname{enc}(A)=B$. However, by Theorem 6.4 , we must have that $|A| \geqslant|B|-d$. Consequently, $v \in \mathcal{C}^{\prime}$. On the other hand, suppose that $C \subset A$. As $\operatorname{enc}(C)=B$ and as $B$ is dead, we must also have that $v \in \mathcal{C}^{\prime}$. Thus, $\mathcal{C}^{\prime}=\mathcal{C}$ as claimed.

Theorem 8.11. The graph $\mathcal{G}$ has finite components of arbitrarily large height and it contains infinitely many isolated vertices.

Proof. Let $\alpha=2 l^{3}+l^{2}+l$ and $\beta=2 l^{3}+l^{2}-l$ for some integer $l \geqslant 3$. Note that $\alpha$ and $\beta$ are always positive. Consider the box $B=B(\alpha, \beta)$. Then $B$ is not congruent to the box $B(m-1, m+1)$ for any odd integer $m$, and $B$ is not congruent to a Wang-Wang box. Therefore by Theorem $8.5, B$ is a dead set.

By Proposition 5.13, we have that $\operatorname{Ex}(B)=l^{3}$. Furthermore, given any standard line $L, B \cap L$ is either empty or contains at least $\min \left(\frac{\alpha}{2}+1, \frac{\beta}{2}+1\right)=l^{3}+\frac{l^{2}}{2}-\frac{l}{2}+1$ vertices. In particular, as $l \geqslant 3, B \cap L$ is either empty or contains at least $l^{3}+2=\operatorname{Ex}(B)+2$ vertices. Thus by Proposition 8.10, the component of $\mathcal{G}$ containing $B$ is finite and has height at least $l^{3}$. As this is true for any $l \geqslant 3$, the first claim follows.

Now let $\alpha=k^{2}+k$ and $\beta=k^{2}-k$ for some integer $k \geqslant 4$. Then we claim that the box $B=B(\alpha, \beta)$ is an isolated vertex of $\mathcal{G}$. By Theorem $5.13, \operatorname{Ex}(B)=0$. As above, for any standard line $L, B \cap L$ is either empty or contains at least 2 vertices. Also $B$ is a dead set by Theorem 8.5. Therefore by Proposition $8.10, B$ is an isolated vertex of $\mathcal{G}$. Thus $\mathcal{G}$ contains infinitely many isolated vertices.

\section{References}

[BE18] Ben Barber and Joshua Erde, Isoperimetry in integer lattices, Discrete Analysis 7 (2018).

[Ber67] A. J. Bernstein, Maximally connected arrays on the $n$-cube, SIAM J. Appl. Math. 15 (1967), 1485-1489.

[Bez99] S. L. Bezrukov, Edge isoperimetric problems on graphs, Graph theory and combinatorial biology (Balatonlelle, 1996), Bolyai Soc. Math. Stud., vol. 7, János Bolyai Math. Soc., Budapest, 1999, pp. 157-197.

[BL91a] Béla Bollobás and Imre Leader, Compressions and isoperimetric inequalities, J. Combin. Theory Ser. A 56 (1991), no. 1, 47-62. 
[BL91b] - Edge-isoperimetric inequalities in the grid, Combinatorica 11 (1991), no. 4, 299-314.

[BL09] Sergei L. Bezrukov and Uwe Leck, A simple proof of the Karakhanyan-Riordan theorem on the even discrete torus, SIAM J. Discrete Math. 23 (2009), no. 3, 1416-1421.

[BS02] Sergei L. Bezrukov and Oriol Serra, A local-global principle for vertexisoperimetric problems, vol. 257, 2002, Kleitman and combinatorics: a celebration (Cambridge, MA, 1999), pp. 285-309.

[Chv75] Jarmila Chvátalová, Optimal labelling of a product of two paths, Discrete Math. 11 (1975), 249-253.

[Har64] L. H. Harper, Optimal assignments of numbers to vertices, J. Soc. Indust. Appl. Math. 12 (1964), 131-135.

[Har66]__ Optimal numberings and isoperimetric problems on graphs, J. Combinatorial Theory 1 (1966), 385-393.

[Har76] Sergiu Hart, A note on the edges of the $n$-cube, Discrete Math. 14 (1976), no. 2, $157-163$.

[Har99] L. H. Harper, On an isoperimetric problem for Hamming graphs, Proceedings of the Conference on Optimal Discrete Structures and Algorithms - ODSA '97 (Rostock), vol. 95, 1999, pp. 285-309.

[Har04] Global methods for combinatorial isoperimetric problems, Cambridge Studies in Advanced Mathematics, vol. 90, Cambridge University Press, 2004.

[HLW06] Shlomo Hoory, Nathan Linial, and Avi Wigderson, Expander graphs and their applications, Bull. Amer. Math. Soc. (N.S.) 43 (2006), no. 4, 439-561.

[Kar82] V. M. Karakhanyan, A discrete isoperimetric problem on a multidimensional torus, Akad. Nauk Armyan. SSR Dokl. 74 (1982), no. 2, 61-65.

[Lin64] John H. Lindsey, II, Assignment of numbers to vertices, Amer. Math. Monthly 71 (1964), 508-516.

[Mog83] H.S. Moghadam, Compression operators and a solution to the bandwidth problem of the product of $n$ paths, Ph.D. Thesis, University of California, Riverside (1983).

[Rio98] Oliver Riordan, An ordering on the even discrete torus, SIAM J. Discrete Math. 11 (1998), no. 1, 110-127.

[Sie08] Nándor Sieben, Polyominoes with minimum site-perimeter and full set achievement games, European J. Combin. 29 (2008), no. 1, 108-117.

[VB08] Daniel Vainsencher and Alfred M. Bruckstein, On isoperimetrically optimal polyforms, Theoret. Comput. Sci. 406 (2008), no. 1-2, 146-159.

[VR12] Ellen Veomett and A. J. Radcliffe, Vertex isoperimetric inequalities for a family of graphs on $\mathbb{Z}^{k}$, Electron. J. Combin. 19(2) (2012), \#P45.

[WW77] Da Lun Wang and Ping Wang, Discrete isoperimetric problems, SIAM J. Appl. Math. 32 (1977), no. 4, 860-870. 